Supporting Information for

\title{
GROUP 13 SUPERACID ADDUCTS OF $\left[\mathrm{PCl}_{2} \mathrm{~N}\right]_{3}$
}

Zin-Min Tun, Amy J. Heston, Matthew J. Panzner, Vincenzo Scionti, Doug A. Medvetz, Brian

D. Wright, Nicholas A. Johnson, Linlin Li, Chrys Wesdemiotis, Peter L. Rinaldi, Wiley J.

Youngs, and Claire A. Tessier*

Department of Chemistry, The University of Akron, OH 44325-3601, USA

tessier@uakron.edu

\begin{tabular}{|c|c|}
\hline & Pages \\
\hline The crystal structure of monoclinic $\left[\mathrm{PCl}_{2} \mathrm{~N}_{3} \cdot \mathrm{HAICl}_{4}\right.$ & $2-7$ \\
\hline The crystal structure of $\left[\mathrm{PCl}_{2} \mathrm{~N}\right]_{3} \cdot \mathrm{HGaCl}_{4}$ & $8-13$ \\
\hline The crystal structure of $\left[\mathrm{PCl}_{2} \mathrm{~N}\right]_{3} \cdot \mathrm{HAIBr}_{4}$ & 14-19 \\
\hline The crystal structure of orthorhombic $\left[\mathrm{PCl}_{2} \mathrm{~N}\right]_{3} \cdot \mathrm{HAICl}_{4}$ & $20-35$ \\
\hline $\begin{array}{l}\text { The reactions of }\left[\mathrm{PCl}_{2} \mathrm{~N}\right]_{3} \text { with } \mathrm{HX} \text { (experimental procedures and } \\
\text { NMR data) }\end{array}$ & $36-37$ \\
\hline The ${ }^{31} \mathrm{P}$ VT NMR data for $\left[\mathrm{PCl}_{2} \mathrm{~N}_{3} \cdot \mathrm{HGaCl}_{4}\right.$ and $\left[\mathrm{PCl}_{2} \mathrm{~N}\right]_{3} \cdot \mathrm{HAIBr}_{4}$ & 38 \\
\hline The ${ }^{1} \mathrm{H}$ VT NMR data for $\left[\mathrm{PCl}_{2} \mathrm{~N}_{3} \cdot \mathrm{HGaCl}_{4}\right.$ and $\left[\mathrm{PCl}_{2} \mathrm{~N}\right]_{3} \cdot \mathrm{HAIBr}_{4}$ & 39 \\
\hline $\begin{array}{l}\text { The }{ }^{1} \mathrm{H} \text { and }{ }^{31} \mathrm{P} \text { VT NMR data for }\left[\mathrm{PCl}_{2} \mathrm{~N}_{3} \cdot \mathrm{HGaCl}_{4} \text { in the presence of }\right. \\
\text { excess } \mathrm{GaCl}_{3}\end{array}$ & 40 \\
\hline The ESI mass spectra for $\left[\mathrm{PCl}_{2} \mathrm{~N}\right]_{3} \cdot \mathrm{HGaCl}_{4}$ and $\left[\mathrm{PCl}_{2} \mathrm{~N}\right]_{3} \cdot \mathrm{HAIBr}_{4}$ & $41-42$ \\
\hline
\end{tabular}


The crystal structure of monoclinic $\left[\mathrm{PCl}_{2} \mathrm{~N}_{3} \mathrm{HAICl}_{4}\right.$
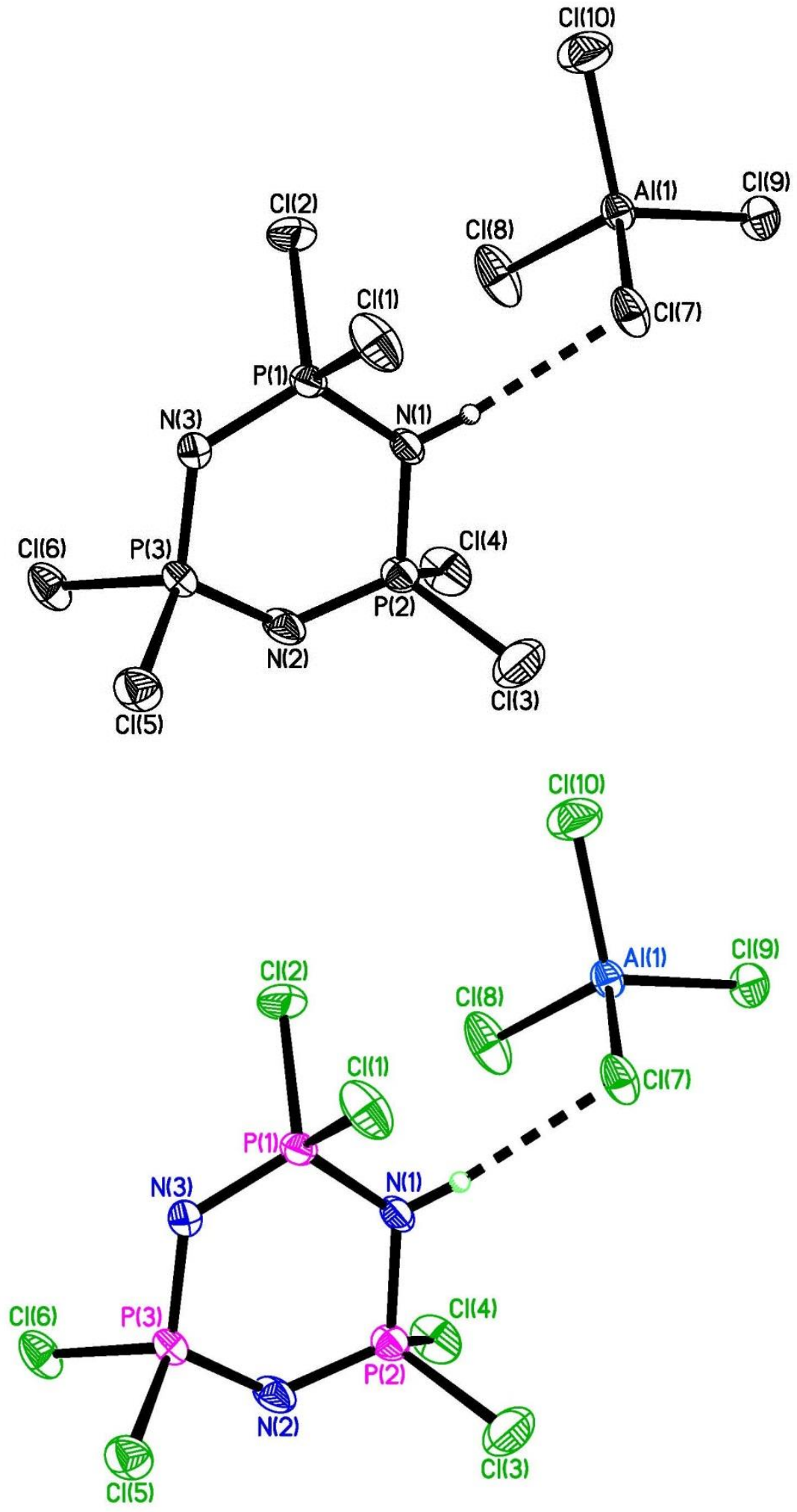
Table 1. Crystal data and structure refinement for monoclinic $\left[\mathrm{PCl}_{2} \mathrm{~N}_{3} \cdot \mathrm{HAICl}_{4}\right.$.

Empirical formula

Formula weight

Temperature

Wavelength

Crystal system

Space group

Unit cell dimensions

Volume

$\mathrm{Z}$

Density (calculated)

Absorption coefficient

$\mathrm{F}(000)$

Crystal size

Theta range for data collection

Index ranges

Reflections collected

Independent reflections

Completeness to theta $=26.30^{\circ}$

Absorption correction

Max. and min. transmission

Refinement method

Data / restraints / parameters

Goodness-of-fit on $\mathrm{F}^{2}$

Final R indices [I $>2 \operatorname{sigma}(\mathrm{I})]$

$\mathrm{R}$ indices (all data)

Largest diff. peak and hole

\section{Al Cl10 H N3 P3}

517.43

100(2) K

$0.71073 \AA$

Monoclinic

$\mathrm{P} 2(1) / \mathrm{n}$

$\mathrm{a}=12.684(3) \AA$

$\alpha=90^{\circ}$.

$\mathrm{b}=10.277(3) \AA$

$\beta=108.495(4)^{\circ}$.

$\mathrm{c}=13.962(4) \AA$

$\gamma=90^{\circ}$.

$1726.0(8) \AA^{3}$

4

$1.991 \mathrm{Mg} / \mathrm{m}^{3}$

$1.923 \mathrm{~mm}^{-1}$

1000

$0.39 \times 0.34 \times 0.07 \mathrm{~mm}^{3}$

1.89 to $26.30^{\circ}$.

$-15<=\mathrm{h}<=15,-12<=\mathrm{k}<=12,-17<=1<=16$

13468

$3505[\mathrm{R}(\mathrm{int})=0.0366]$

$100.0 \%$

Semi-empirical from equivalents

0.8771 and 0.5209

Full-matrix least-squares on $\mathrm{F}^{2}$

$3505 / 0 / 158$

1.663

$\mathrm{R} 1=0.0288, w \mathrm{R} 2=0.0635$

$\mathrm{R} 1=0.0322, \mathrm{wR} 2=0.0643$

0.557 and -0.501 e. $\AA^{-3}$ 
Table 2. Atomic coordinates ( $\mathrm{x} 10^{4}$ ) and equivalent isotropic displacement parameters $\left(\AA^{2} \times 10^{3}\right)$ for $\left[\mathrm{PCl}_{2} \mathrm{~N}_{3}\right]_{3} \mathrm{HAICl}_{4}$. U(eq) is defined as one third of the trace of the orthogonalized $\mathrm{U}^{\mathrm{ij}}$ tensor.

\begin{tabular}{lcccc}
\hline & $\mathrm{x}$ & $\mathrm{y}$ & $\mathrm{z}$ & $\mathrm{U}(\mathrm{eq})$ \\
\hline $\mathrm{Cl}(1)$ & $10466(1)$ & $2627(1)$ & $3612(1)$ & $32(1)$ \\
$\mathrm{Cl}(2)$ & $10603(1)$ & $5062(1)$ & $2379(1)$ & $33(1)$ \\
$\mathrm{Cl}(3)$ & $8693(1)$ & $74(1)$ & $873(1)$ & $41(1)$ \\
$\mathrm{Cl}(4)$ & $8603(1)$ & $2165(1)$ & $-741(1)$ & $37(1)$ \\
$\mathrm{Cl}(5)$ & $6608(1)$ & $2358(1)$ & $2465(1)$ & $26(1)$ \\
$\mathrm{Cl}(6)$ & $6277(1)$ & $4795(1)$ & $1081(1)$ & $30(1)$ \\
$\mathrm{Cl}(7)$ & $2936(1)$ & $6726(1)$ & $3341(1)$ & $28(1)$ \\
$\mathrm{Cl}(8)$ & $3550(1)$ & $9178(1)$ & $5091(1)$ & $46(1)$ \\
$\mathrm{Cl}(9)$ & $1292(1)$ & $7071(1)$ & $4896(1)$ & $30(1)$ \\
$\mathrm{Cl}(10)$ & $1118(1)$ & $9317(1)$ & $2991(1)$ & $46(1)$ \\
$\mathrm{P}(1)$ & $9640(1)$ & $3578(1)$ & $2379(1)$ & $18(1)$ \\
$\mathrm{P}(2)$ & $8528(1)$ & $1960(1)$ & $629(1)$ & $21(1)$ \\
$\mathrm{P}(3)$ & $7330(1)$ & $3372(1)$ & $1645(1)$ & $18(1)$ \\
$\mathrm{Al}(1)$ & $8114(1)$ & $4107(1)$ & $21(1)$ \\
$\mathrm{N}(1)$ & $2198(1)$ & $2657(2)$ & $1409(1)$ & $21(1)$ \\
$\mathrm{N}(2)$ & $9639(2)$ & $2494(2)$ & $747(1)$ & $26(1)$ \\
$\mathrm{N}(3)$ & $7437(2)$ & $2361(1)$ & $20(1)$ \\
\hline & $8458(1)$ & & \\
\hline
\end{tabular}


Table 3. Bond lengths $[\AA]$ and angles $\left[{ }^{\circ}\right]$ for $\left[\mathrm{PCl}_{2} \mathrm{~N}_{3} \cdot \mathrm{HAICl}_{4}\right.$.

\begin{tabular}{|c|c|}
\hline $\mathrm{Cl}(1)-\mathrm{P}(1)$ & $1.9665(9)$ \\
\hline $\mathrm{Cl}(2)-\mathrm{P}(1)$ & $1.9538(9)$ \\
\hline $\mathrm{Cl}(3)-\mathrm{P}(2)$ & $1.9683(10)$ \\
\hline $\mathrm{Cl}(4)-\mathrm{P}(2)$ & $1.9559(9)$ \\
\hline $\mathrm{Cl}(5)-\mathrm{P}(3)$ & $1.9746(8)$ \\
\hline $\mathrm{Cl}(6)-\mathrm{P}(3)$ & $1.9678(8)$ \\
\hline $\mathrm{Cl}(7)-\mathrm{Al}(1)$ & $2.1646(9)$ \\
\hline $\mathrm{Cl}(8)-\mathrm{Al}(1)$ & $2.1286(10)$ \\
\hline $\mathrm{Cl}(9)-\mathrm{Al}(1)$ & $2.1186(9)$ \\
\hline $\mathrm{Cl}(10)-\mathrm{Al}(1)$ & $2.1165(10)$ \\
\hline $\mathrm{P}(1)-\mathrm{N}(3)$ & $1.5438(18)$ \\
\hline $\mathrm{P}(1)-\mathrm{N}(1)$ & $1.6524(19)$ \\
\hline $\mathrm{P}(2)-\mathrm{N}(2)$ & $1.5458(19)$ \\
\hline $\mathrm{P}(2)-\mathrm{N}(1)$ & $1.6461(19)$ \\
\hline $\mathrm{P}(3)-\mathrm{N}(3)$ & $1.5844(18)$ \\
\hline $\mathrm{P}(3)-\mathrm{N}(2)$ & $1.5854(19)$ \\
\hline $\mathrm{N}(3)-\mathrm{P}(1)-\mathrm{N}(1)$ & $112.68(10)$ \\
\hline $\mathrm{N}(3)-\mathrm{P}(1)-\mathrm{Cl}(2)$ & $113.76(8)$ \\
\hline $\mathrm{N}(1)-\mathrm{P}(1)-\mathrm{Cl}(2)$ & $106.56(7)$ \\
\hline $\mathrm{N}(3)-\mathrm{P}(1)-\mathrm{Cl}(1)$ & $112.78(8)$ \\
\hline $\mathrm{N}(1)-\mathrm{P}(1)-\mathrm{Cl}(1)$ & $107.12(8)$ \\
\hline $\mathrm{Cl}(2)-\mathrm{P}(1)-\mathrm{Cl}(1)$ & $103.22(4)$ \\
\hline $\mathrm{N}(2)-\mathrm{P}(2)-\mathrm{N}(1)$ & $112.51(10)$ \\
\hline $\mathrm{N}(2)-\mathrm{P}(2)-\mathrm{Cl}(4)$ & $112.89(8)$ \\
\hline $\mathrm{N}(1)-\mathrm{P}(2)-\mathrm{Cl}(4)$ & 107.67(8) \\
\hline $\mathrm{N}(2)-\mathrm{P}(2)-\mathrm{Cl}(3)$ & $112.37(8)$ \\
\hline $\mathrm{N}(1)-\mathrm{P}(2)-\mathrm{Cl}(3)$ & $106.97(8)$ \\
\hline $\mathrm{Cl}(4)-\mathrm{P}(2)-\mathrm{Cl}(3)$ & $103.87(4)$ \\
\hline $\mathrm{N}(3)-\mathrm{P}(3)-\mathrm{N}(2)$ & $115.53(10)$ \\
\hline $\mathrm{N}(3)-\mathrm{P}(3)-\mathrm{Cl}(6)$ & $109.24(7)$ \\
\hline $\mathrm{N}(2)-\mathrm{P}(3)-\mathrm{Cl}(6)$ & $108.90(8)$ \\
\hline $\mathrm{N}(3)-\mathrm{P}(3)-\mathrm{Cl}(5)$ & 109.19(7) \\
\hline $\mathrm{N}(2)-\mathrm{P}(3)-\mathrm{Cl}(5)$ & $109.26(8)$ \\
\hline
\end{tabular}




$\begin{array}{ll}\mathrm{Cl}(6)-\mathrm{P}(3)-\mathrm{Cl}(5) & 104.11(4) \\ \mathrm{Cl}(10)-\mathrm{Al}(1)-\mathrm{Cl}(9) & 110.31(4) \\ \mathrm{Cl}(10)-\mathrm{Al}(1)-\mathrm{Cl}(8) & 112.06(5) \\ \mathrm{Cl}(9)-\mathrm{Al}(1)-\mathrm{Cl}(8) & 112.52(4) \\ \mathrm{Cl}(10)-\mathrm{Al}(1)-\mathrm{Cl}(7) & 107.58(4) \\ \mathrm{Cl}(9)-\mathrm{Al}(1)-\mathrm{Cl}(7) & 108.33(4) \\ \mathrm{Cl}(8)-\mathrm{Al}(1)-\mathrm{Cl}(7) & 105.74(4) \\ \mathrm{P}(2)-\mathrm{N}(1)-\mathrm{P}(1) & 124.72(12) \\ \mathrm{P}(2)-\mathrm{N}(2)-\mathrm{P}(3) & 126.20(12) \\ \mathrm{P}(1)-\mathrm{N}(3)-\mathrm{P}(3) & 126.15(12)\end{array}$

Symmetry transformations used to generate equivalent atoms:

Table 4. Anisotropic displacement parameters $\left(\AA^{2} \times 10^{3}\right)$ for $\left[\mathrm{PCl}_{2} \mathrm{~N}\right]_{3} \cdot \mathrm{HAICl}_{4}$. The anisotropic displacement factor exponent takes the form: $-2 \pi^{2}\left[h^{2} a^{* 2} U^{11}+\ldots+2 h k a^{*} b^{*} U^{12}\right]$

\begin{tabular}{lcccccc}
\hline & $\mathrm{U}^{11}$ & $\mathrm{U}^{22}$ & $\mathrm{U}^{33}$ & $\mathrm{U}^{23}$ & $\mathrm{U}^{13}$ & $\mathrm{U}^{12}$ \\
\hline $\mathrm{Cl}(1)$ & $22(1)$ & $52(1)$ & $22(1)$ & $10(1)$ & $6(1)$ & $9(1)$ \\
$\mathrm{Cl}(2)$ & $27(1)$ & $29(1)$ & $43(1)$ & $-7(1)$ & $13(1)$ & $-12(1)$ \\
$\mathrm{Cl}(3)$ & $48(1)$ & $24(1)$ & $48(1)$ & $-7(1)$ & $12(1)$ & $-4(1)$ \\
$\mathrm{Cl}(4)$ & $33(1)$ & $60(1)$ & $19(1)$ & $-4(1)$ & $11(1)$ & $-2(1)$ \\
$\mathrm{Cl}(5)$ & $26(1)$ & $26(1)$ & $30(1)$ & $5(1)$ & $13(1)$ & $-2(1)$ \\
$\mathrm{Cl}(6)$ & $20(1)$ & $33(1)$ & $39(1)$ & $14(1)$ & $10(1)$ & $8(1)$ \\
$\mathrm{Cl}(7)$ & $22(1)$ & $31(1)$ & $36(1)$ & $-14(1)$ & $16(1)$ & $-8(1)$ \\
$\mathrm{Cl}(8)$ & $34(1)$ & $59(1)$ & $51(1)$ & $-34(1)$ & $20(1)$ & $-20(1)$ \\
$\mathrm{Cl}(9)$ & $31(1)$ & $34(1)$ & $30(1)$ & $4(1)$ & $18(1)$ & $-1(1)$ \\
$\mathrm{Cl}(10)$ & $40(1)$ & $53(1)$ & $53(1)$ & $29(1)$ & $25(1)$ & $17(1)$ \\
$\mathrm{P}(1)$ & $14(1)$ & $21(1)$ & $18(1)$ & $-2(1)$ & $4(1)$ & $-2(1)$ \\
$\mathrm{P}(2)$ & $18(1)$ & $27(1)$ & $18(1)$ & $-4(1)$ & $5(1)$ & $-2(1)$ \\
$\mathrm{P}(3)$ & $14(1)$ & $22(1)$ & $20(1)$ & $2(1)$ & $5(1)$ & $1(1)$ \\
$\mathrm{Al}(1)$ & $19(1)$ & $24(1)$ & $22(1)$ & $-2(1)$ & $10(1)$ & $-2(1)$ \\
$\mathrm{N}(1)$ & $13(1)$ & $28(1)$ & $23(1)$ & $-5(1)$ & $6(1)$ & $1(1)$ \\
$\mathrm{N}(2)$ & $15(1)$ & $39(1)$ & $22(1)$ & $-9(1)$ & $3(1)$ & $-4(1)$ \\
$\mathrm{N}(3)$ & $17(1)$ & $21(1)$ & $23(1)$ & $-5(1)$ & $7(1)$ & $0(1)$ \\
& & & & & & \\
\hline
\end{tabular}


Table 5. Hydrogen coordinates ( x 104) and isotropic displacement parameters $\left(\AA^{2} \mathrm{x} 10^{3}\right)$ for $\left[\mathrm{PCl}_{2} \mathrm{~N}\right]_{3} \cdot \mathrm{HAICl}_{4}$.

\begin{tabular}{lcccc}
\hline & $\mathrm{x}$ & $\mathrm{y}$ & $\mathrm{z}$ & $\mathrm{U}(\mathrm{eq})$ \\
\hline $\mathrm{H}(1)$ & & & & \\
\hline & $10270(20)$ & $2380(30)$ & $1410(20)$ & $44(8)$ \\
\hline
\end{tabular}

Table 6. Hydrogen bonds for $\left[\mathrm{PCl}_{2} \mathrm{~N}\right]_{3} \cdot \mathrm{HAICl}_{4}\left[\AA\right.$ and $\left.{ }^{\circ}\right]$.

\begin{tabular}{lcccc}
\hline $\mathrm{D}-\mathrm{H} \ldots \mathrm{A}$ & $\mathrm{d}(\mathrm{D}-\mathrm{H})$ & $\mathrm{d}(\mathrm{H} \ldots \mathrm{A})$ & $\mathrm{d}(\mathrm{D} \ldots \mathrm{A})$ & $<(\mathrm{DHA})$ \\
\hline $\mathrm{N}(1)-\mathrm{H}(1) \ldots \mathrm{Cl}(7) \# 1$ & $0.85(3)$ & $2.29(3)$ & $3.133(2)$ & $171(2)$ \\
\hline
\end{tabular}

Symmetry transformations used to generate equivalent atoms:

$\# 1-\mathrm{x}+3 / 2, \mathrm{y}-1 / 2,-\mathrm{z}+1 / 2$ 
The crystal structure of $\left[\mathrm{PCl}_{2} \mathrm{~N}\right]_{3} \mathrm{HGaCl}_{4}$
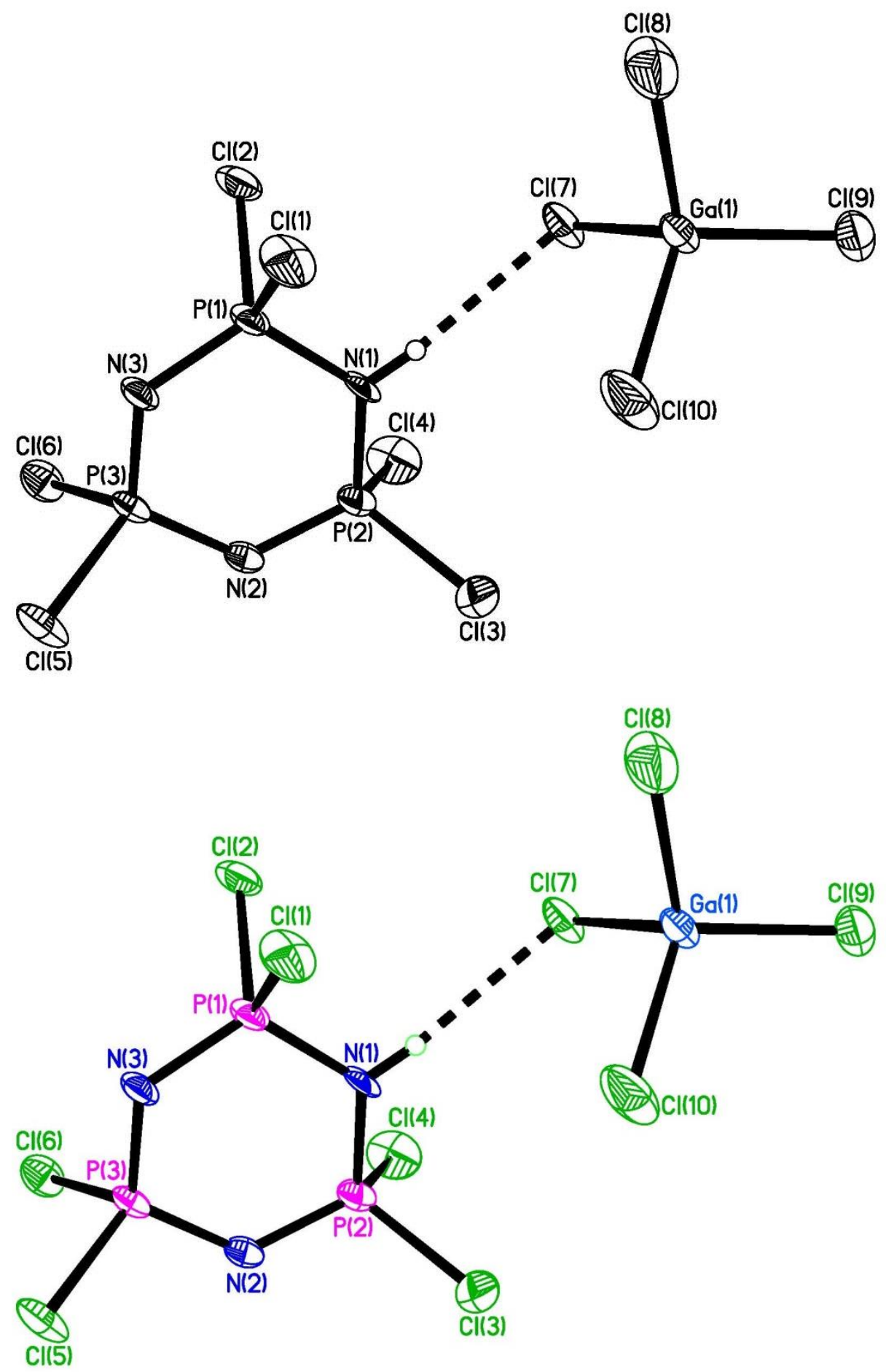
Table 1. Crystal data and structure refinement for $\left[\mathrm{PCl}_{2} \mathrm{~N}_{3} \cdot \mathrm{HGaCl}_{4}\right.$.

Empirical formula

Formula weight

Temperature

Wavelength

Crystal system

Space group

Unit cell dimensions

Volume

$\mathrm{Z}$

Density (calculated)

Absorption coefficient

$\mathrm{F}(000)$

Crystal size

Theta range for data collection

Index ranges

Reflections collected

Independent reflections

Completeness to theta $=26.30^{\circ}$

Absorption correction

Max. and min. transmission

Refinement method

Data / restraints / parameters

Goodness-of-fit on $\mathrm{F}^{2}$

Final $\mathrm{R}$ indices [I>2sigma(I)]

$\mathrm{R}$ indices (all data)

Largest diff. peak and hole

\section{Cl10 Ga H N3 P3}

560.17

100(2) K

$0.71073 \AA$

Monoclinic

$\mathrm{P} 2(1) / \mathrm{n}$

$\mathrm{a}=12.723(5) \AA$

$\alpha=90^{\circ}$.

$\mathrm{b}=10.263(4) \AA$

$\beta=108.603(6)^{\circ}$.

$\mathrm{c}=13.981(6) \AA$

$\gamma=90^{\circ}$.

4

$2.150 \mathrm{Mg} / \mathrm{m}^{3}$

$3.391 \mathrm{~mm}^{-1}$

1072

$0.29 \times 0.10 \times 0.06 \mathrm{~mm}^{3}$

1.89 to $26.30^{\circ}$.

$-15<=\mathrm{h}<=15,-12<=\mathrm{k}<=12,-17<=1<=16$

13033

$3500[\mathrm{R}(\mathrm{int})=0.0648]$

$99.7 \%$

Semi-empirical from equivalents

0.8224 and 0.4396

Full-matrix least-squares on $\mathrm{F}^{2}$

$3500 / 0 / 158$

0.939

$\mathrm{R} 1=0.0366, \mathrm{wR} 2=0.0815$

$\mathrm{R} 1=0.0485, \mathrm{wR} 2=0.0830$

0.813 and -1.088 e. $\AA^{-3}$ 
Table 2. Atomic coordinates ( $\left.\times 10^{4}\right)$ and equivalent isotropic displacement parameters $\left(\AA^{2} \times 10^{3}\right)$ for $\left[\mathrm{PCl}_{2} \mathrm{~N}_{3} \mathrm{HGaCl}_{4}\right.$. U(eq) is defined as one third of the trace of the orthogonalized $\mathrm{U}^{\mathrm{ij}}$ tensor.

\begin{tabular}{lcccc}
\hline & $\mathrm{x}$ & $\mathrm{y}$ & $\mathrm{z}$ & $\mathrm{U}(\mathrm{eq})$ \\
\hline $\mathrm{Ga}(1)$ & $7191(1)$ & $6905(1)$ & $9113(1)$ & $23(1)$ \\
$\mathrm{Cl}(1)$ & $-603(1)$ & $4933(1)$ & $7626(1)$ & $35(1)$ \\
$\mathrm{Cl}(2)$ & $-469(1)$ & $7373(1)$ & $6394(1)$ & $34(1)$ \\
$\mathrm{Cl}(3)$ & $1393(1)$ & $7840(1)$ & $10744(1)$ & $38(1)$ \\
$\mathrm{Cl}(4)$ & $1303(1)$ & $9932(1)$ & $9126(1)$ & $42(1)$ \\
$\mathrm{Cl}(5)$ & $3712(1)$ & $5194(1)$ & $8928(1)$ & $33(1)$ \\
$\mathrm{Cl}(6)$ & $3392(1)$ & $7639(1)$ & $7549(1)$ & $28(1)$ \\
$\mathrm{Cl}(7)$ & $7948(1)$ & $8316(1)$ & $8334(1)$ & $29(1)$ \\
$\mathrm{Cl}(8)$ & $6086(1)$ & $5689(1)$ & $7973(1)$ & $48(1)$ \\
$\mathrm{Cl}(9)$ & $6276(1)$ & $7974(1)$ & $9916(1)$ & $32(1)$ \\
$\mathrm{Cl}(10)$ & $8574(1)$ & $5816(1)$ & $10104(1)$ & $50(1)$ \\
$\mathrm{P}(1)$ & $355(1)$ & $6423(1)$ & $7625(1)$ & $20(1)$ \\
$\mathrm{P}(2)$ & $1467(1)$ & $8042(1)$ & $9374(1)$ & $23(1)$ \\
$\mathrm{P}(3)$ & $2666(1)$ & $6626(1)$ & $8364(1)$ & $21(1)$ \\
$\mathrm{N}(1)$ & $359(2)$ & $7347(3)$ & $8594(2)$ & $24(1)$ \\
$\mathrm{N}(2)$ & $2560(2)$ & $7501(3)$ & $9259(2)$ & $26(1)$ \\
$\mathrm{N}(3)$ & $1545(2)$ & $6037(3)$ & $7645(2)$ & $22(1)$ \\
& & & & \\
\hline
\end{tabular}


Table 3. Bond lengths $[\AA]$ and angles $\left[{ }^{\circ}\right]$ for $\left[\mathrm{PCl}_{2} \mathrm{~N}_{3} \cdot \mathrm{HGaCl}_{4}\right.$.

\begin{tabular}{|c|c|}
\hline $\mathrm{Ga}(1)-\mathrm{Cl}(8)$ & $2.1549(13)$ \\
\hline $\mathrm{Ga}(1)-\mathrm{Cl}(9)$ & $2.1582(11)$ \\
\hline $\mathrm{Ga}(1)-\mathrm{Cl}(10)$ & $2.1700(12)$ \\
\hline $\mathrm{Ga}(1)-\mathrm{Cl}(7)$ & $2.2095(11)$ \\
\hline $\mathrm{Cl}(1)-\mathrm{P}(1)$ & $1.9556(14)$ \\
\hline $\mathrm{Cl}(2)-\mathrm{P}(1)$ & $1.9649(14)$ \\
\hline $\mathrm{Cl}(3)-\mathrm{P}(2)$ & $1.9577(16)$ \\
\hline $\mathrm{Cl}(4)-\mathrm{P}(2)$ & $1.9698(16)$ \\
\hline $\mathrm{Cl}(5)-\mathrm{P}(3)$ & $1.9710(13)$ \\
\hline $\mathrm{Cl}(6)-\mathrm{P}(3)$ & $1.9756(14)$ \\
\hline $\mathrm{P}(1)-\mathrm{N}(3)$ & $1.556(3)$ \\
\hline $\mathrm{P}(1)-\mathrm{N}(1)$ & $1.652(3)$ \\
\hline $\mathrm{P}(2)-\mathrm{N}(2)$ & $1.554(3)$ \\
\hline $\mathrm{P}(2)-\mathrm{N}(1)$ & $1.644(3)$ \\
\hline $\mathrm{P}(3)-\mathrm{N}(3)$ & $1.580(3)$ \\
\hline $\mathrm{P}(3)-\mathrm{N}(2)$ & $1.580(3)$ \\
\hline $\mathrm{Cl}(8)-\mathrm{Ga}(1)-\mathrm{Cl}(9)$ & $110.23(5)$ \\
\hline $\mathrm{Cl}(8)-\mathrm{Ga}(1)-\mathrm{Cl}(10)$ & $112.27(6)$ \\
\hline $\mathrm{Cl}(9)-\mathrm{Ga}(1)-\mathrm{Cl}(10)$ & $113.04(5)$ \\
\hline $\mathrm{Cl}(8)-\mathrm{Ga}(1)-\mathrm{Cl}(7)$ & $107.43(5)$ \\
\hline $\mathrm{Cl}(9)-\mathrm{Ga}(1)-\mathrm{Cl}(7)$ & $108.43(5)$ \\
\hline $\mathrm{Cl}(10)-\mathrm{Ga}(1)-\mathrm{Cl}(7)$ & $105.09(5)$ \\
\hline $\mathrm{N}(3)-\mathrm{P}(1)-\mathrm{N}(1)$ & $112.50(16)$ \\
\hline $\mathrm{N}(3)-\mathrm{P}(1)-\mathrm{Cl}(1)$ & $113.83(12)$ \\
\hline $\mathrm{N}(1)-\mathrm{P}(1)-\mathrm{Cl}(1)$ & $106.67(12)$ \\
\hline $\mathrm{N}(3)-\mathrm{P}(1)-\mathrm{Cl}(2)$ & $112.69(12)$ \\
\hline $\mathrm{N}(1)-\mathrm{P}(1)-\mathrm{Cl}(2)$ & $107.16(13)$ \\
\hline $\mathrm{Cl}(1)-\mathrm{P}(1)-\mathrm{Cl}(2)$ & $103.32(6)$ \\
\hline $\mathrm{N}(2)-\mathrm{P}(2)-\mathrm{N}(1)$ & $112.47(17)$ \\
\hline $\mathrm{N}(2)-\mathrm{P}(2)-\mathrm{Cl}(3)$ & $112.76(13)$ \\
\hline $\mathrm{N}(1)-\mathrm{P}(2)-\mathrm{Cl}(3)$ & $107.73(13)$ \\
\hline $\mathrm{N}(2)-\mathrm{P}(2)-\mathrm{Cl}(4)$ & $112.47(13)$ \\
\hline $\mathrm{N}(1)-\mathrm{P}(2)-\mathrm{Cl}(4)$ & $106.88(13)$ \\
\hline
\end{tabular}




$\begin{array}{ll}\mathrm{Cl}(3)-\mathrm{P}(2)-\mathrm{Cl}(4) & 103.99(6) \\ \mathrm{N}(3)-\mathrm{P}(3)-\mathrm{N}(2) & 115.73(15) \\ \mathrm{N}(3)-\mathrm{P}(3)-\mathrm{Cl}(5) & 109.06(12) \\ \mathrm{N}(2)-\mathrm{P}(3)-\mathrm{Cl}(5) & 108.88(13) \\ \mathrm{N}(3)-\mathrm{P}(3)-\mathrm{Cl}(6) & 109.14(12) \\ \mathrm{N}(2)-\mathrm{P}(3)-\mathrm{Cl}(6) & 109.32(13) \\ \mathrm{Cl}(5)-\mathrm{P}(3)-\mathrm{Cl}(6) & 104.07(6) \\ \mathrm{P}(2)-\mathrm{N}(1)-\mathrm{P}(1) & 124.83(19) \\ \mathrm{P}(2)-\mathrm{N}(2)-\mathrm{P}(3) & 126.2(2) \\ \mathrm{P}(1)-\mathrm{N}(3)-\mathrm{P}(3) & 126.10(19)\end{array}$

Symmetry transformations used to generate equivalent atoms:

Table 4. Anisotropic displacement parameters $\left(\AA^{2} \times 10^{3}\right)$ for $\left[\mathrm{PCl}_{2} \mathrm{~N}_{3} \cdot \mathrm{HGaCl}_{4}\right.$. The anisotropic displacement factor exponent takes the form: $-2 \pi^{2}\left[h^{2} a^{* 2} U^{11}+\ldots+2 h k a^{*} b^{*} U^{12}\right]$

\begin{tabular}{lcccccc}
\hline & $\mathrm{U}^{11}$ & $\mathrm{U}^{22}$ & $\mathrm{U}^{33}$ & $\mathrm{U}^{23}$ & $\mathrm{U}^{13}$ & $\mathrm{U}^{12}$ \\
\hline $\mathrm{Ga}(1)$ & $13(1)$ & $27(1)$ & $28(1)$ & $3(1)$ & $7(1)$ & $2(1)$ \\
$\mathrm{Cl}(1)$ & $21(1)$ & $33(1)$ & $49(1)$ & $-7(1)$ & $10(1)$ & $-12(1)$ \\
$\mathrm{Cl}(2)$ & $16(1)$ & $56(1)$ & $27(1)$ & $9(1)$ & $1(1)$ & $9(1)$ \\
$\mathrm{Cl}(3)$ & $27(1)$ & $62(1)$ & $25(1)$ & $-4(1)$ & $7(1)$ & $-1(1)$ \\
$\mathrm{Cl}(4)$ & $41(1)$ & $28(1)$ & $52(1)$ & $-6(1)$ & $7(1)$ & $-4(1)$ \\
$\mathrm{Cl}(5)$ & $15(1)$ & $37(1)$ & $44(1)$ & $14(1)$ & $7(1)$ & $9(1)$ \\
$\mathrm{Cl}(6)$ & $20(1)$ & $29(1)$ & $36(1)$ & $5(1)$ & $10(1)$ & $-2(1)$ \\
$\mathrm{Cl}(7)$ & $16(1)$ & $34(1)$ & $40(1)$ & $13(1)$ & $12(1)$ & $8(1)$ \\
$\mathrm{Cl}(8)$ & $34(1)$ & $55(1)$ & $61(1)$ & $-29(1)$ & $22(1)$ & $-18(1)$ \\
$\mathrm{Cl}(9)$ & $26(1)$ & $39(1)$ & $35(1)$ & $-4(1)$ & $15(1)$ & $0(1)$ \\
$\mathrm{Cl}(10)$ & $27(1)$ & $64(1)$ & $60(1)$ & $37(1)$ & $18(1)$ & $20(1)$ \\
$\mathrm{P}(1)$ & $8(1)$ & $24(1)$ & $24(1)$ & $-2(1)$ & $1(1)$ & $-2(1)$ \\
$\mathrm{P}(2)$ & $13(1)$ & $30(1)$ & $23(1)$ & $-4(1)$ & $1(1)$ & $-2(1)$ \\
$\mathrm{P}(3)$ & $9(1)$ & $25(1)$ & $25(1)$ & $2(1)$ & $2(1)$ & $1(1)$ \\
$\mathrm{N}(1)$ & $4(2)$ & $34(2)$ & $29(2)$ & $-8(1)$ & $0(1)$ & $0(1)$ \\
$\mathrm{N}(2)$ & $10(2)$ & $40(2)$ & $24(2)$ & $-7(1)$ & $-1(1)$ & $-3(1)$ \\
$\mathrm{N}(3)$ & $9(2)$ & $26(2)$ & $27(2)$ & $-6(1)$ & $1(1)$ & $1(1)$ \\
& & & & & & \\
\hline
\end{tabular}


Table 5. Hydrogen coordinates ( x $\left.10^{4}\right)$ and isotropic displacement parameters $\left(\AA^{2} \times 10^{3}\right)$ for $\left[\mathrm{PCl}_{2} \mathrm{~N}\right]_{3} \cdot \mathrm{HGaCl}_{4}$.

\begin{tabular}{lcccc}
\hline & $x$ & $y$ & $z$ & $U(e q)$ \\
\hline$H(1)$ & $-230(30)$ & $7610(40)$ & $8570(30)$ & $26(11)$ \\
\hline
\end{tabular}

Table 6. Hydrogen bonds for $\left[\left[\mathrm{PCl}_{2} \mathrm{~N}_{3} \cdot \mathrm{HGaCl}_{4}\left[\AA\right.\right.\right.$ and $\left.{ }^{\circ}\right]$.

\begin{tabular}{lcccc}
\hline $\mathrm{D}-\mathrm{H} \ldots \mathrm{A}$ & $\mathrm{d}(\mathrm{D}-\mathrm{H})$ & $\mathrm{d}(\mathrm{H} \ldots \mathrm{A})$ & $\mathrm{d}(\mathrm{D} \ldots \mathrm{A})$ & $<(\mathrm{DHA})$ \\
\hline $\mathrm{N}(1)-\mathrm{H}(1) \ldots \mathrm{Cl}(7) \# 1$ & $0.78(4)$ & $2.35(4)$ & $3.134(3)$ & $174(4)$ \\
\hline
\end{tabular}

Symmetry transformations used to generate equivalent atoms:

$\# 1 \mathrm{x}-1, \mathrm{y}, \mathrm{z}$ 
The crystal structure of $\left[\mathrm{PCl}_{2} \mathrm{~N}\right]_{3} \cdot \mathrm{HAIBr}_{4}$
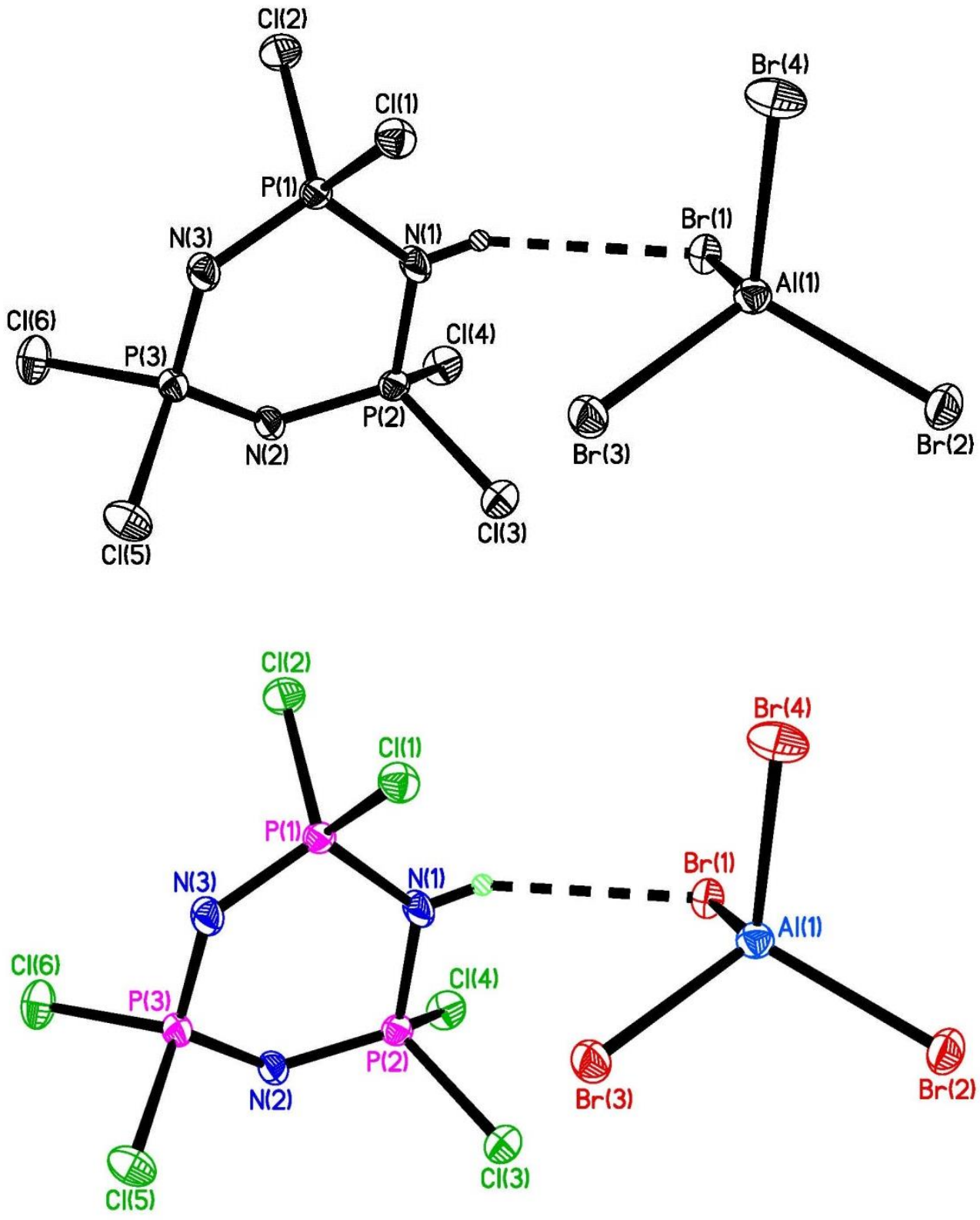
Table 1. Crystal data and structure refinement for $\left[\mathrm{PCl}_{2} \mathrm{~N}_{3} \mathrm{HAIBr}_{4}\right.$.

Empirical formula

Formula weight

Temperature

Wavelength

Crystal system

Space group

Unit cell dimensions

Volume

$\mathrm{Z}$

Density (calculated)

Absorption coefficient

$\mathrm{F}(000)$

Crystal size

Theta range for data collection

Index ranges

Reflections collected

Independent reflections

Completeness to theta $=26.30^{\circ}$

Absorption correction

Max. and min. transmission

Refinement method

Data / restraints / parameters

Goodness-of-fit on $\mathrm{F}^{2}$

Final $\mathrm{R}$ indices [I $>2 \operatorname{sigma}(\mathrm{I})]$

$\mathrm{R}$ indices (all data)

Largest diff. peak and hole
Al Br4 Cl6 H N3 P3

695.27

100(2) K

$0.71073 \AA$

Triclinic

P-1

$a=7.6721(9) \AA$ $\alpha=84.643(2)^{\circ}$.

$\mathrm{b}=9.9263(12) \AA$ $\beta=85.055(2)^{\circ}$.

$\mathrm{c}=12.3245(14) \AA$ $\gamma=73.178(2)^{\circ}$.

892.77(18) $\AA^{3}$

2

$2.586 \mathrm{Mg} / \mathrm{m}^{3}$

$10.212 \mathrm{~mm}^{-1}$

644

$0.10 \times 0.06 \times 0.03 \mathrm{~mm}^{3}$

1.66 to $26.30^{\circ}$.

$-9<=\mathrm{h}<=9,-12<=\mathrm{k}<=12,-14<=\mathrm{l}<=15$

7222

$3598[\mathrm{R}(\mathrm{int})=0.0240]$

$98.9 \%$

Semi-empirical from equivalents

0.7492 and 0.4283

Full-matrix least-squares on $\mathrm{F}^{2}$

3598 / 0 / 158

1.026

$\mathrm{R} 1=0.0308, \mathrm{wR} 2=0.0637$

$\mathrm{R} 1=0.0395, \mathrm{wR} 2=0.0663$

0.892 and -0.511 e. $\AA^{-3}$ 
Table 2. Atomic coordinates ( $\mathrm{x} 10^{4}$ ) and equivalent isotropic displacement parameters $\left(\AA^{2} \times 10^{3}\right)$ for $\left[\mathrm{PCl}_{2} \mathrm{~N}_{3} \cdot \mathrm{HAIBr}_{4}\right.$. U(eq) is defined as one third of the trace of the orthogonalized $\mathrm{U}^{\mathrm{ij}}$ tensor.

\begin{tabular}{lrrrr}
\hline & $\mathrm{x}$ & $\mathrm{y}$ & $\mathrm{z}$ & $\mathrm{U}(\mathrm{eq})$ \\
\hline $\mathrm{Br}(1)$ & $3145(1)$ & $5232(1)$ & $3948(1)$ & $17(1)$ \\
$\mathrm{Br}(2)$ & $54(1)$ & $8753(1)$ & $3041(1)$ & $23(1)$ \\
$\mathrm{Br}(3)$ & $4731(1)$ & $7444(1)$ & $1621(1)$ & $21(1)$ \\
$\mathrm{Br}(4)$ & $4267(1)$ & $8495(1)$ & $4509(1)$ & $25(1)$ \\
$\mathrm{Cl}(1)$ & $8570(1)$ & $5442(1)$ & $3220(1)$ & $19(1)$ \\
$\mathrm{Cl}(2)$ & $10382(1)$ & $2342(1)$ & $4072(1)$ & $22(1)$ \\
$\mathrm{Cl}(3)$ & $4367(1)$ & $3818(1)$ & $1195(1)$ & $22(1)$ \\
$\mathrm{Cl}(4)$ & $5434(1)$ & $933(1)$ & $2542(1)$ & $23(1)$ \\
$\mathrm{Cl}(5)$ & $9766(2)$ & $3510(1)$ & $-599(1)$ & $24(1)$ \\
$\mathrm{Cl}(6)$ & $11950(1)$ & $709(1)$ & $571(1)$ & $25(1)$ \\
$\mathrm{P}(1)$ & $9085(1)$ & $3487(1)$ & $2847(1)$ & $14(1)$ \\
$\mathrm{P}(2)$ & $6456(1)$ & $2419(1)$ & $1855(1)$ & $15(1)$ \\
$\mathrm{P}(3)$ & $9838(1)$ & $2359(1)$ & $805(1)$ & $14(1)$ \\
$\mathrm{Al}(1)$ & $3048(2)$ & $7509(1)$ & $3259(1)$ & $16(1)$ \\
$\mathrm{N}(1)$ & $3170(4)$ & $2838(3)$ & $21(1)$ \\
$\mathrm{N}(2)$ & $1847(4)$ & $1008(3)$ & $17(1)$ \\
$\mathrm{N}(3)$ & $3232(4)$ & $1731(3)$ & $18(1)$ \\
& & & & \\
\hline
\end{tabular}


Table 3. Bond lengths $[\AA]$ and angles $\left[{ }^{\circ}\right]$ for $\left[\mathrm{PCl}_{2} \mathrm{~N}\right]_{3} \cdot \mathrm{HAIBr}_{4}$.

\begin{tabular}{|c|c|}
\hline $\operatorname{Br}(1)-\operatorname{Al}(1)$ & $2.3212(13)$ \\
\hline $\operatorname{Br}(2)-\operatorname{Al}(1)$ & $2.2971(13)$ \\
\hline $\operatorname{Br}(3)-\operatorname{Al}(1)$ & $2.2963(13)$ \\
\hline $\operatorname{Br}(4)-\operatorname{Al}(1)$ & $2.2949(13)$ \\
\hline $\mathrm{Cl}(1)-\mathrm{P}(1)$ & $1.9555(15)$ \\
\hline $\mathrm{Cl}(2)-\mathrm{P}(1)$ & $1.9607(15)$ \\
\hline $\mathrm{Cl}(3)-\mathrm{P}(2)$ & $1.9706(15)$ \\
\hline $\mathrm{Cl}(4)-\mathrm{P}(2)$ & $1.9565(15)$ \\
\hline $\mathrm{Cl}(5)-\mathrm{P}(3)$ & $1.9777(15)$ \\
\hline $\mathrm{Cl}(6)-\mathrm{P}(3)$ & $1.9658(15)$ \\
\hline $\mathrm{P}(1)-\mathrm{N}(3)$ & $1.554(3)$ \\
\hline $\mathrm{P}(1)-\mathrm{N}(1)$ & $1.658(4)$ \\
\hline $\mathrm{P}(2)-\mathrm{N}(2)$ & $1.555(3)$ \\
\hline $\mathrm{P}(2)-\mathrm{N}(1)$ & $1.652(4)$ \\
\hline $\mathrm{P}(3)-\mathrm{N}(2)$ & $1.577(3)$ \\
\hline $\mathrm{P}(3)-\mathrm{N}(3)$ & $1.582(3)$ \\
\hline $\mathrm{N}(3)-\mathrm{P}(1)-\mathrm{N}(1)$ & 111.71(18) \\
\hline $\mathrm{N}(3)-\mathrm{P}(1)-\mathrm{Cl}(1)$ & $111.86(14)$ \\
\hline $\mathrm{N}(1)-\mathrm{P}(1)-\mathrm{Cl}(1)$ & $106.34(15)$ \\
\hline $\mathrm{N}(3)-\mathrm{P}(1)-\mathrm{Cl}(2)$ & $113.07(15)$ \\
\hline $\mathrm{N}(1)-\mathrm{P}(1)-\mathrm{Cl}(2)$ & $108.24(15)$ \\
\hline $\mathrm{Cl}(1)-\mathrm{P}(1)-\mathrm{Cl}(2)$ & $105.18(6)$ \\
\hline $\mathrm{N}(2)-\mathrm{P}(2)-\mathrm{N}(1)$ & 111.94(18) \\
\hline $\mathrm{N}(2)-\mathrm{P}(2)-\mathrm{Cl}(4)$ & $112.26(14)$ \\
\hline $\mathrm{N}(1)-\mathrm{P}(2)-\mathrm{Cl}(4)$ & $107.59(14)$ \\
\hline $\mathrm{N}(2)-\mathrm{P}(2)-\mathrm{Cl}(3)$ & $112.93(14)$ \\
\hline $\mathrm{N}(1)-\mathrm{P}(2)-\mathrm{Cl}(3)$ & $107.36(15)$ \\
\hline $\mathrm{Cl}(4)-\mathrm{P}(2)-\mathrm{Cl}(3)$ & $104.30(7)$ \\
\hline $\mathrm{N}(2)-\mathrm{P}(3)-\mathrm{N}(3)$ & $115.27(18)$ \\
\hline $\mathrm{N}(2)-\mathrm{P}(3)-\mathrm{Cl}(6)$ & 108.67(14) \\
\hline $\mathrm{N}(3)-\mathrm{P}(3)-\mathrm{Cl}(6)$ & $110.26(14)$ \\
\hline $\mathrm{N}(2)-\mathrm{P}(3)-\mathrm{Cl}(5)$ & $110.06(14)$ \\
\hline $\mathrm{N}(3)-\mathrm{P}(3)-\mathrm{Cl}(5)$ & $108.44(14)$ \\
\hline
\end{tabular}




$\begin{array}{ll}\mathrm{Cl}(6)-\mathrm{P}(3)-\mathrm{Cl}(5) & 103.50(7) \\ \mathrm{Br}(4)-\mathrm{Al}(1)-\operatorname{Br}(3) & 109.99(5) \\ \mathrm{Br}(4)-\mathrm{Al}(1)-\operatorname{Br}(2) & 110.47(5) \\ \operatorname{Br}(3)-\mathrm{Al}(1)-\operatorname{Br}(2) & 110.64(5) \\ \operatorname{Br}(4)-\mathrm{Al}(1)-\operatorname{Br}(1) & 107.41(5) \\ \operatorname{Br}(3)-\operatorname{Al}(1)-\operatorname{Br}(1) & 109.93(5) \\ \operatorname{Br}(2)-\mathrm{Al}(1)-\operatorname{Br}(1) & 108.32(5) \\ \mathrm{P}(2)-\mathrm{N}(1)-\mathrm{P}(1) & 124.7(2) \\ \mathrm{P}(2)-\mathrm{N}(2)-\mathrm{P}(3) & 125.5(2) \\ \mathrm{P}(1)-\mathrm{N}(3)-\mathrm{P}(3) & 126.3(2)\end{array}$

Symmetry transformations used to generate equivalent atoms:

Table 4. Anisotropic displacement parameters $\left(\AA^{2} \times 10^{3}\right)$ for $\left[\mathrm{PCl}_{2} \mathrm{~N}\right]_{3} \cdot \mathrm{HAlBr}_{4}$. The anisotropic displacement factor exponent takes the form: $-2 \pi^{2}\left[h^{2} a^{* 2} U^{11}+\ldots+2 h k a^{*} b^{*} U^{12}\right]$

\begin{tabular}{lcccccc}
\hline & $\mathrm{U}^{11}$ & $\mathrm{U}^{22}$ & $\mathrm{U}^{33}$ & $\mathrm{U}^{23}$ & $\mathrm{U}^{13}$ & $\mathrm{U}^{12}$ \\
\hline $\mathrm{Br}(1)$ & $15(1)$ & $15(1)$ & $20(1)$ & $0(1)$ & $1(1)$ & $-3(1)$ \\
$\mathrm{Br}(2)$ & $18(1)$ & $22(1)$ & $24(1)$ & $1(1)$ & $-1(1)$ & $2(1)$ \\
$\mathrm{Br}(3)$ & $20(1)$ & $22(1)$ & $16(1)$ & $1(1)$ & $2(1)$ & $-2(1)$ \\
$\mathrm{Br}(4)$ & $37(1)$ & $27(1)$ & $18(1)$ & $-1(1)$ & $-5(1)$ & $-19(1)$ \\
$\mathrm{Cl}(1)$ & $21(1)$ & $16(1)$ & $19(1)$ & $-3(1)$ & $-1(1)$ & $-5(1)$ \\
$\mathrm{Cl}(2)$ & $23(1)$ & $22(1)$ & $18(1)$ & $3(1)$ & $-5(1)$ & $-4(1)$ \\
$\mathrm{Cl}(3)$ & $19(1)$ & $23(1)$ & $22(1)$ & $0(1)$ & $-3(1)$ & $-2(1)$ \\
$\mathrm{Cl}(4)$ & $22(1)$ & $24(1)$ & $25(1)$ & $4(1)$ & $-1(1)$ & $-13(1)$ \\
$\mathrm{Cl}(5)$ & $32(1)$ & $26(1)$ & $16(1)$ & $3(1)$ & $0(1)$ & $-10(1)$ \\
$\mathrm{Cl}(6)$ & $18(1)$ & $19(1)$ & $34(1)$ & $-5(1)$ & $3(1)$ & $0(1)$ \\
$\mathrm{P}(1)$ & $13(1)$ & $18(1)$ & $13(1)$ & $-2(1)$ & $-1(1)$ & $-7(1)$ \\
$\mathrm{P}(2)$ & $13(1)$ & $17(1)$ & $15(1)$ & $-1(1)$ & $-1(1)$ & $-6(1)$ \\
$\mathrm{P}(3)$ & $14(1)$ & $16(1)$ & $13(1)$ & $-1(1)$ & $1(1)$ & $-4(1)$ \\
$\mathrm{Al}(1)$ & $17(1)$ & $15(1)$ & $15(1)$ & $-1(1)$ & $-2(1)$ & $-4(1)$ \\
$\mathrm{N}(1)$ & $15(2)$ & $36(2)$ & $17(2)$ & $-9(2)$ & $6(2)$ & $-13(2)$ \\
$\mathrm{N}(2)$ & $15(2)$ & $20(2)$ & $15(2)$ & $-6(2)$ & $1(1)$ & $-5(2)$ \\
$\mathrm{N}(3)$ & $15(2)$ & $23(2)$ & $18(2)$ & $-4(2)$ & $3(2)$ & $-10(2)$ \\
& & & & & & \\
\hline
\end{tabular}


Table 5. Hydrogen coordinates ( x 104) and isotropic displacement parameters $\left(\AA^{2} \mathrm{x} 10^{3}\right)$ for $\left[\mathrm{PCl}_{2} \mathrm{~N}_{3} \cdot \mathrm{HAIBr}_{4}\right.$.

\begin{tabular}{lcccc}
\hline & $\mathrm{x}$ & $\mathrm{y}$ & $\mathrm{z}$ & $\mathrm{U}(\mathrm{eq})$ \\
\hline $\mathrm{H}(1)$ & & & & \\
\hline
\end{tabular}

Table 6. Hydrogen bonds for $\left[\mathrm{PCl}_{2} \mathrm{~N}\right]_{3} \cdot \mathrm{HAIBr}_{4}\left[\AA\right.$ And $\left.^{\circ}\right]$.

\begin{tabular}{lcccc}
\hline $\mathrm{D}-\mathrm{H} \ldots \mathrm{A}$ & $\mathrm{d}(\mathrm{D}-\mathrm{H})$ & $\mathrm{d}(\mathrm{H} \ldots \mathrm{A})$ & $\mathrm{d}(\mathrm{D} \ldots \mathrm{A})$ & $<(\mathrm{DHA})$ \\
\hline $\mathrm{N}(1)-\mathrm{H}(1) \ldots \mathrm{Br}(1)$ & $0.80(5)$ & $2.65(5)$ & $3.378(4)$ & $151(5)$ \\
\hline
\end{tabular}

Symmetry transformations used to generate equivalent atoms: 
The crystal structure of orthorhombic $\left[\mathrm{PCl}_{2} \mathrm{~N}\right]_{3} \cdot \mathrm{HAICl}_{4}$

The overall asymmetric unit and asymmetric units units 1 and 2 that show the disorder model

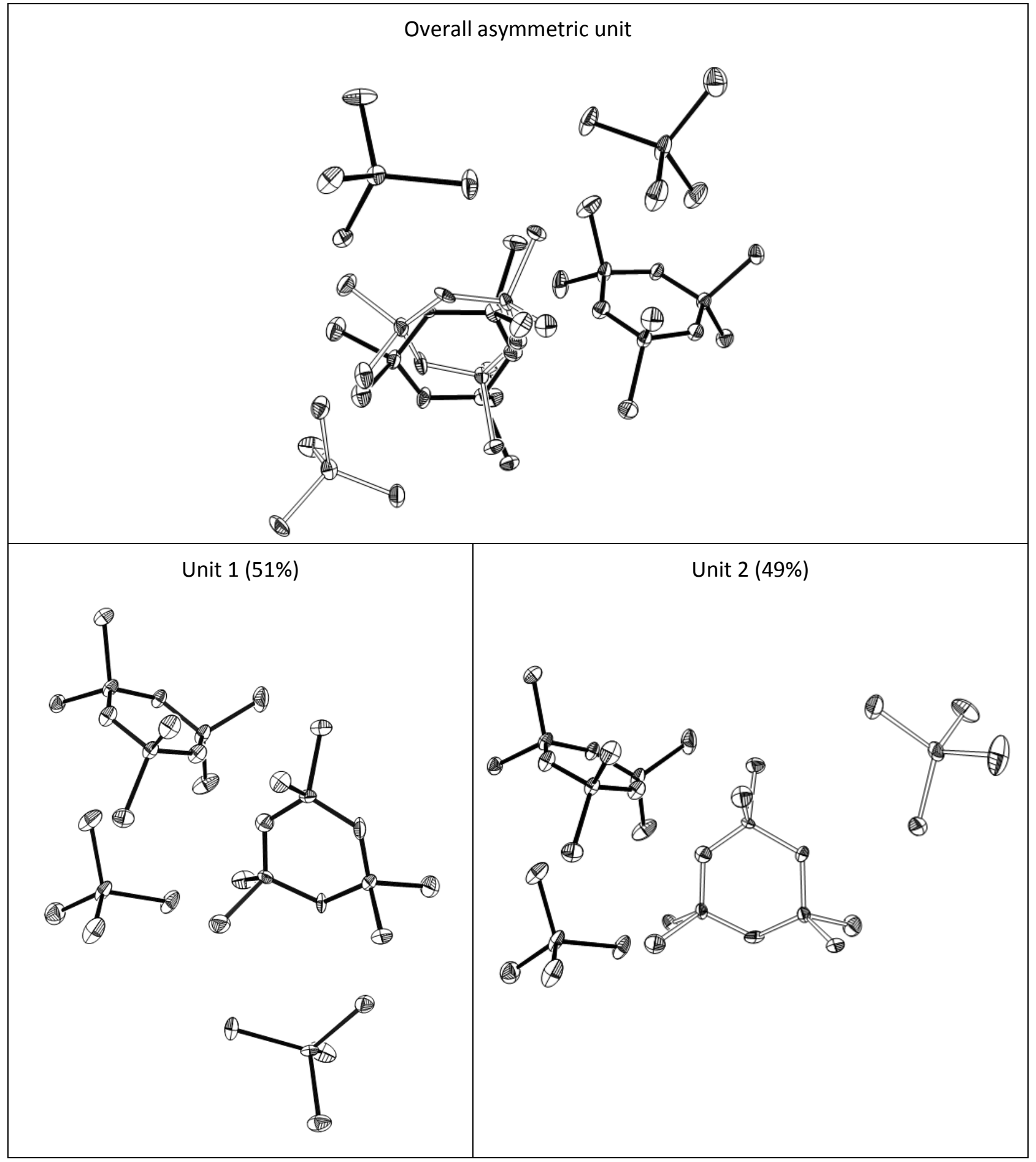


Table 1. Crystal data and structure refinement for orthorhombic $\left[\mathrm{PCl}_{2} \mathrm{~N}_{3} \cdot \mathrm{HAICl}_{4}\right.$.

Empirical formula

Formula weight

Temperature

Wavelength

Crystal system

Space group

Unit cell dimensions

Volume

Z

Density (calculated)

Absorption coefficient

$\mathrm{F}(000)$

Crystal size

Theta range for data collection

Index ranges

Reflections collected

Independent reflections

Completeness to theta $=26.30^{\circ}$

Absorption correction

\section{Al Cl10 N3 P3}

516.42

100(2) K

$0.71073 \AA$

Orthorhombic

P2(1)2(1)2(1)

$\mathrm{a}=10.545(3) \AA \quad \alpha=90^{\circ}$.

$\mathrm{b}=10.619(3) \AA \quad \beta=90^{\circ}$.

$\mathrm{c}=29.920(8) \AA \quad \gamma=90^{\circ}$.

$3350.2(15) \AA^{3}$

8

$2.048 \mathrm{Mg} / \mathrm{m}^{3}$

$1.981 \mathrm{~mm}^{-1}$

1992

$0.23 \times 0.15 \times 0.05 \mathrm{~mm}^{3}$

1.36 to $26.30^{\circ}$.

$-13 \leq \mathrm{h} \leq 12,-13 \leq \mathrm{k} \leq 13,-37 \leq 1 \leq 37$

26839

$6800[\mathrm{R}(\mathrm{int})=0.0467]$

$99.8 \%$

Semi-empirical from equivalents 
Max. and min. transmission

Refinement method

Data / restraints / parameters

Goodness-of-fit on $\mathrm{F}^{2}$

Final $R$ indices [I $>2 \operatorname{sigma}(\mathrm{I})]$

$\mathrm{R}$ indices (all data)

Absolute structure parameter

Largest diff. peak and hole
0.9074 and 0.6586

Full-matrix least-squares on $\mathrm{F}^{2}$

$6800 / 0 / 453$

1.197

$\mathrm{R} 1=0.0617, \mathrm{wR} 2=0.1614$

$\mathrm{R} 1=0.0643, \mathrm{wR} 2=0.1629$

0.61(19)

1.118 and -0.488 e. $\AA^{-3}$ 
Table 2. Atomic coordinates ( $\times 10^{4}$ ) and equivalent isotropic displacement parameters $\left(\AA^{2} \times 10^{3}\right)$

for orthorhombic $\left[\mathrm{PCl}_{2} \mathrm{~N}_{3} \cdot \mathrm{HAICl}\right.$. $\mathrm{U}(\mathrm{eq})$ is defined as one third of the trace of the orthogonalized $\mathrm{U}^{\mathrm{ij}}$ tensor.

\begin{tabular}{|c|c|c|c|c|}
\hline & $\mathrm{x}$ & $\mathrm{y}$ & z & $\mathrm{U}(\mathrm{eq})$ \\
\hline $\mathrm{Cl}(1)$ & 4031(2) & $5305(2)$ & $1698(1)$ & $26(1)$ \\
\hline $\mathrm{Cl}(2)$ & $1110(2)$ & $5261(2)$ & $1726(1)$ & $28(1)$ \\
\hline $\mathrm{Cl}(3)$ & $4141(2)$ & $3160(2)$ & 3181(1) & $32(1)$ \\
\hline $\mathrm{Cl}(4)$ & $1214(2)$ & $3012(2)$ & $3168(1)$ & $31(1)$ \\
\hline $\mathrm{Cl}(5)$ & $4248(2)$ & $7578(2)$ & $3116(1)$ & $43(1)$ \\
\hline $\mathrm{Cl}(6)$ & $1345(2)$ & 7881(2) & $3129(1)$ & $42(1)$ \\
\hline $\mathrm{P}(1)$ & $2604(2)$ & $5248(1)$ & $2123(1)$ & $21(1)$ \\
\hline $\mathrm{P}(2)$ & $2624(2)$ & $4060(1)$ & 2961(1) & $20(1)$ \\
\hline $\mathrm{P}(3)$ & $2670(2)$ & $6702(1)$ & $2954(1)$ & $25(1)$ \\
\hline $\mathrm{N}(1)$ & $2658(6)$ & $4068(5)$ & $2430(2)$ & $24(1)$ \\
\hline $\mathrm{N}(2)$ & $2550(6)$ & $5402(4)$ & $3183(2)$ & $24(1)$ \\
\hline $\mathrm{N}(3)$ & $2617(6)$ & $6588(5)$ & $2403(2)$ & $22(1)$ \\
\hline $\mathrm{Cl}(7)$ & $6179(2)$ & $5307(2)$ & $2576(1)$ & $44(1)$ \\
\hline $\mathrm{Cl}(8)$ & $7647(3)$ & $3596(2)$ & $3407(1)$ & $45(1)$ \\
\hline $\mathrm{Cl}(9)$ & 7492(3) & $6880(2)$ & $3506(1)$ & $40(1)$ \\
\hline $\mathrm{Cl}(10)$ & $9422(2)$ & $5499(2)$ & 2694(1) & $44(1)$ \\
\hline $\mathrm{Al}(1)$ & $7679(2)$ & $5362(2)$ & $3046(1)$ & $31(1)$ \\
\hline $\mathrm{Cl}(11)$ & 1832(4) & 6497(4) & $5593(1)$ & $35(1)$ \\
\hline
\end{tabular}




\begin{tabular}{|c|c|c|c|c|}
\hline $\mathrm{Cl}(12)$ & $2264(6)$ & $3642(5)$ & 5693(2) & $28(1)$ \\
\hline $\mathrm{Cl}(13)$ & $-209(5)$ & 5414(4) & 4072(1) & $27(1)$ \\
\hline $\mathrm{Cl}(14)$ & $276(5)$ & 2631(5) & $4330(2)$ & $25(1)$ \\
\hline $\mathrm{Cl}(15)$ & $4757(4)$ & $5763(4)$ & 4161(1) & $31(1)$ \\
\hline $\mathrm{Cl}(16)$ & $4576(5)$ & $3008(5)$ & $4485(2)$ & $32(1)$ \\
\hline $\mathrm{P}(4 \mathrm{~A})$ & 2035(3) & 4961(3) & $5236(1)$ & $21(1)$ \\
\hline $\mathrm{P}(5 \mathrm{~A})$ & $965(5)$ & $4354(5)$ & $4412(2)$ & $20(1)$ \\
\hline $\mathrm{P}(6 \mathrm{~A})$ & $3599(5)$ & $4582(4)$ & $4450(1)$ & $18(1)$ \\
\hline $\mathrm{N}(4 \mathrm{~A})$ & $3399(12)$ & $5042(10)$ & 4970(5) & $17(2)$ \\
\hline $\mathrm{N}(5 \mathrm{~A})$ & $901(12)$ & $4639(14)$ & 4930(4) & $30(3)$ \\
\hline $\mathrm{N}(6 \mathrm{~A})$ & $2336(16)$ & $4427(13)$ & 4193(4) & $24(3)$ \\
\hline $\mathrm{Cl}(17)$ & $3206(4)$ & $6562(4)$ & $5549(1)$ & $35(1)$ \\
\hline $\mathrm{Cl}(18)$ & $2850(6)$ & $3699(5)$ & $5693(2)$ & $29(1)$ \\
\hline $\mathrm{Cl}(19)$ & $424(5)$ & $5767(4)$ & $4120(1)$ & $25(1)$ \\
\hline $\mathrm{Cl}(20)$ & $516(5)$ & $3016(5)$ & $4446(2)$ & $28(1)$ \\
\hline $\mathrm{Cl}(21)$ & $5357(4)$ & $5349(4)$ & 4064(1) & $23(1)$ \\
\hline $\mathrm{Cl}(22)$ & $4850(4)$ & $2592(5)$ & $4339(2)$ & $28(1)$ \\
\hline $\mathrm{P}(4 \mathrm{~B})$ & $3054(4)$ & 4999(4) & $5219(2)$ & $22(1)$ \\
\hline $\mathrm{P}(5 \mathrm{~B})$ & $1516(5)$ & $4563(5)$ & $4425(1)$ & $16(1)$ \\
\hline $\mathrm{P}(6 \mathrm{~B})$ & 4154(5) & 4311(4) & $4408(1)$ & $16(1)$ \\
\hline $\mathrm{N}(4 \mathrm{~B})$ & $4189(12)$ & $4733(13)$ & 4920(4) & $20(2)$ \\
\hline $\mathrm{N}(5 \mathrm{~B})$ & $1684(13)$ & $5078(14)$ & 4951(4) & $26(3)$ \\
\hline $\mathrm{N}(6 \mathrm{~B})$ & 2790(16) & $4344(13)$ & 4190(5) & $22(3)$ \\
\hline
\end{tabular}




\begin{tabular}{lcccc}
$\mathrm{Cl}(23)$ & $8077(4)$ & $4432(4)$ & $6135(1)$ & $33(1)$ \\
$\mathrm{Cl}(24)$ & $8222(4)$ & $7299(4)$ & $5590(2)$ & $41(1)$ \\
$\mathrm{Cl}(25)$ & $5305(3)$ & $5829(3)$ & $5759(1)$ & $26(1)$ \\
$\mathrm{Cl}(26)$ & $7684(15)$ & $4450(19)$ & $4959(7)$ & $37(2)$ \\
$\mathrm{Al}(2 \mathrm{~A})$ & $7301(6)$ & $5522(8)$ & $5605(3)$ & $20(1)$ \\
$\mathrm{Cl}(27)$ & $-2983(6)$ & $4524(5)$ & $6123(1)$ & $53(2)$ \\
$\mathrm{Cl}(28)$ & $-3149(4)$ & $7272(4)$ & $5526(2)$ & $42(1)$ \\
$\mathrm{Cl}(29)$ & $-223(3)$ & $5856(4)$ & $5714(1)$ & $31(1)$ \\
$\mathrm{Cl}(30)$ & $-2623(15)$ & $4634(19)$ & $4993(7)$ & $39(2)$ \\
$\mathrm{Al}(2 \mathrm{~B})$ & $-2197(7)$ & $5509(9)$ & $5581(3)$ & $24(2)$ \\
& & & & \\
\hline
\end{tabular}


Table 3. Bond lengths $[\AA]$ and angles $\left[{ }^{\circ}\right]$ for orthorhombic $\left[\mathrm{PCl}_{2} \mathrm{~N}\right]_{3} \cdot \mathrm{HAICl}_{4}$.

\begin{tabular}{|c|c|}
\hline $\mathrm{Cl}(1)-\mathrm{P}(1)$ & $1.972(2)$ \\
\hline $\mathrm{Cl}(2)-\mathrm{P}(1)$ & $1.974(2)$ \\
\hline $\mathrm{Cl}(3)-\mathrm{P}(2)$ & $1.976(3)$ \\
\hline $\mathrm{Cl}(4)-\mathrm{P}(2)$ & $1.958(3)$ \\
\hline $\mathrm{Cl}(5)-\mathrm{P}(3)$ & $1.966(3)$ \\
\hline $\mathrm{Cl}(6)-\mathrm{P}(3)$ & $1.948(3)$ \\
\hline $\mathrm{P}(1)-\mathrm{N}(1)$ & $1.554(5)$ \\
\hline $\mathrm{P}(1)-\mathrm{N}(3)$ & $1.650(5)$ \\
\hline $\mathrm{P}(2)-\mathrm{N}(2)$ & $1.576(5)$ \\
\hline $\mathrm{P}(2)-\mathrm{N}(1)$ & $1.588(5)$ \\
\hline $\mathrm{P}(3)-\mathrm{N}(2)$ & $1.546(5)$ \\
\hline $\mathrm{P}(3)-\mathrm{N}(3)$ & $1.656(5)$ \\
\hline $\mathrm{Cl}(7)-\mathrm{Al}(1)$ & $2.117(3)$ \\
\hline $\mathrm{Cl}(8)-\mathrm{Al}(1)$ & $2.166(3)$ \\
\hline $\mathrm{Cl}(9)-\mathrm{Al}(1)$ & $2.130(3)$ \\
\hline $\mathrm{Cl}(10)-\mathrm{Al}(1)$ & $2.123(3)$ \\
\hline $\mathrm{Cl}(11)-\mathrm{P}(4 \mathrm{~A})$ & $1.960(5)$ \\
\hline $\mathrm{Cl}(12)-\mathrm{P}(4 \mathrm{~A})$ & $1.973(6)$ \\
\hline $\mathrm{Cl}(13)-\mathrm{P}(5 \mathrm{~A})$ & $1.957(6)$ \\
\hline $\mathrm{Cl}(14)-\mathrm{P}(5 \mathrm{~A})$ & $1.984(7)$ \\
\hline $\mathrm{Cl}(15)-\mathrm{P}(6 \mathrm{~A})$ & $1.951(5)$ \\
\hline
\end{tabular}




\begin{tabular}{|c|c|}
\hline $\mathrm{Cl}(16)-\mathrm{P}(6 \mathrm{~A})$ & $1.966(7)$ \\
\hline $\mathrm{P}(4 \mathrm{~A})-\mathrm{N}(5 \mathrm{~A})$ & $1.543(13)$ \\
\hline $\mathrm{P}(4 \mathrm{~A})-\mathrm{N}(4 \mathrm{~A})$ & $1.646(15)$ \\
\hline $\mathrm{P}(5 \mathrm{~A})-\mathrm{N}(5 \mathrm{~A})$ & $1.582(12)$ \\
\hline $\mathrm{P}(5 \mathrm{~A})-\mathrm{N}(6 \mathrm{~A})$ & $1.589(16)$ \\
\hline $\mathrm{P}(6 \mathrm{~A})-\mathrm{N}(6 \mathrm{~A})$ & $1.546(16)$ \\
\hline $\mathrm{P}(6 \mathrm{~A})-\mathrm{N}(4 \mathrm{~A})$ & $1.644(14)$ \\
\hline $\mathrm{Cl}(17)-\mathrm{P}(4 \mathrm{~B})$ & $1.939(6)$ \\
\hline $\mathrm{Cl}(18)-\mathrm{P}(4 \mathrm{~B})$ & $1.991(7)$ \\
\hline Cl(19)-P(5B) & $1.946(6)$ \\
\hline $\mathrm{Cl}(20)-\mathrm{P}(5 \mathrm{~B})$ & $1.953(8)$ \\
\hline $\mathrm{Cl}(21)-\mathrm{P}(6 \mathrm{~B})$ & $1.970(5)$ \\
\hline $\mathrm{Cl}(22)-\mathrm{P}(6 \mathrm{~B})$ & $1.978(7)$ \\
\hline $\mathrm{P}(4 \mathrm{~B})-\mathrm{N}(4 \mathrm{~B})$ & $1.520(13)$ \\
\hline $\mathrm{P}(4 \mathrm{~B})-\mathrm{N}(5 \mathrm{~B})$ & $1.654(14)$ \\
\hline $\mathrm{P}(5 \mathrm{~B})-\mathrm{N}(6 \mathrm{~B})$ & $1.534(16)$ \\
\hline $\mathrm{P}(5 \mathrm{~B})-\mathrm{N}(5 \mathrm{~B})$ & $1.677(14)$ \\
\hline$P(6 B)-N(6 B)$ & $1.578(16)$ \\
\hline $\mathrm{P}(6 \mathrm{~B})-\mathrm{N}(4 \mathrm{~B})$ & $1.598(12)$ \\
\hline $\mathrm{Cl}(23)-\mathrm{Al}(2 \mathrm{~A})$ & $2.127(8)$ \\
\hline $\mathrm{Cl}(24)-\mathrm{Al}(2 \mathrm{~A})$ & $2.123(9)$ \\
\hline $\mathrm{Cl}(25)-\mathrm{Al}(2 \mathrm{~A})$ & $2.179(7)$ \\
\hline $\mathrm{Cl}(26)-\mathrm{Al}(2 \mathrm{~A})$ & 2.279(19) \\
\hline
\end{tabular}




\begin{tabular}{|c|c|}
\hline $\mathrm{Cl}(27)-\mathrm{Al}(2 \mathrm{~B})$ & $2.102(10)$ \\
\hline $\mathrm{Cl}(28)-\mathrm{Al}(2 \mathrm{~B})$ & $2.131(9)$ \\
\hline $\mathrm{Cl}(29)-\mathrm{Al}(2 \mathrm{~B})$ & $2.150(9)$ \\
\hline $\mathrm{Cl}(30)-\mathrm{Al}(2 \mathrm{~B})$ & $2.04(2)$ \\
\hline $\mathrm{N}(1)-\mathrm{P}(1)-\mathrm{N}(3)$ & $113.3(3)$ \\
\hline $\mathrm{N}(1)-\mathrm{P}(1)-\mathrm{Cl}(1)$ & $112.2(3)$ \\
\hline $\mathrm{N}(3)-\mathrm{P}(1)-\mathrm{Cl}(1)$ & $107.1(2)$ \\
\hline $\mathrm{N}(1)-\mathrm{P}(1)-\mathrm{Cl}(2)$ & $113.0(3)$ \\
\hline $\mathrm{N}(3)-\mathrm{P}(1)-\mathrm{Cl}(2)$ & $107.8(2)$ \\
\hline $\mathrm{Cl}(1)-\mathrm{P}(1)-\mathrm{Cl}(2)$ & $102.69(9)$ \\
\hline $\mathrm{N}(2)-\mathrm{P}(2)-\mathrm{N}(1)$ & $114.7(3)$ \\
\hline $\mathrm{N}(2)-\mathrm{P}(2)-\mathrm{Cl}(4)$ & $110.0(2)$ \\
\hline $\mathrm{N}(1)-\mathrm{P}(2)-\mathrm{Cl}(4)$ & $109.7(3)$ \\
\hline $\mathrm{N}(2)-\mathrm{P}(2)-\mathrm{Cl}(3)$ & $109.7(2)$ \\
\hline $\mathrm{N}(1)-\mathrm{P}(2)-\mathrm{Cl}(3)$ & $108.5(3)$ \\
\hline $\mathrm{Cl}(4)-\mathrm{P}(2)-\mathrm{Cl}(3)$ & $103.55(10)$ \\
\hline $\mathrm{N}(2)-\mathrm{P}(3)-\mathrm{N}(3)$ & $111.9(3)$ \\
\hline $\mathrm{N}(2)-\mathrm{P}(3)-\mathrm{Cl}(6)$ & $113.4(3)$ \\
\hline $\mathrm{N}(3)-\mathrm{P}(3)-\mathrm{Cl}(6)$ & $106.8(2)$ \\
\hline $\mathrm{N}(2)-\mathrm{P}(3)-\mathrm{Cl}(5)$ & $112.5(3)$ \\
\hline $\mathrm{N}(3)-\mathrm{P}(3)-\mathrm{Cl}(5)$ & $108.0(3)$ \\
\hline $\mathrm{Cl}(6)-\mathrm{P}(3)-\mathrm{Cl}(5)$ & $103.71(13)$ \\
\hline
\end{tabular}




\begin{tabular}{|c|c|}
\hline $\mathrm{P}(1)-\mathrm{N}(1)-\mathrm{P}(2)$ & $126.5(3)$ \\
\hline $\mathrm{P}(3)-\mathrm{N}(2)-\mathrm{P}(2)$ & $128.0(3)$ \\
\hline $\mathrm{P}(1)-\mathrm{N}(3)-\mathrm{P}(3)$ & $124.7(3)$ \\
\hline $\mathrm{Cl}(7)-\mathrm{Al}(1)-\mathrm{Cl}(10)$ & $108.63(12)$ \\
\hline $\mathrm{Cl}(7)-\mathrm{Al}(1)-\mathrm{Cl}(9)$ & $112.42(14)$ \\
\hline $\mathrm{Cl}(10)-\mathrm{Al}(1)-\mathrm{Cl}(9)$ & $110.44(14)$ \\
\hline $\mathrm{Cl}(7)-\mathrm{Al}(1)-\mathrm{Cl}(8)$ & $107.24(13)$ \\
\hline $\mathrm{Cl}(10)-\mathrm{Al}(1)-\mathrm{Cl}(8)$ & $108.69(14)$ \\
\hline $\mathrm{Cl}(9)-\mathrm{Al}(1)-\mathrm{Cl}(8)$ & 109.31(11) \\
\hline $\mathrm{N}(5 \mathrm{~A})-\mathrm{P}(4 \mathrm{~A})-\mathrm{N}(4 \mathrm{~A})$ & $113.7(6)$ \\
\hline $\mathrm{N}(5 \mathrm{~A})-\mathrm{P}(4 \mathrm{~A})-\mathrm{Cl}(11)$ & $115.0(5)$ \\
\hline $\mathrm{N}(4 \mathrm{~A})-\mathrm{P}(4 \mathrm{~A})-\mathrm{Cl}(11)$ & $108.4(4)$ \\
\hline $\mathrm{N}(5 \mathrm{~A})-\mathrm{P}(4 \mathrm{~A})-\mathrm{Cl}(12)$ & $110.4(6)$ \\
\hline $\mathrm{N}(4 \mathrm{~A})-\mathrm{P}(4 \mathrm{~A})-\mathrm{Cl}(12)$ & $105.4(5)$ \\
\hline $\mathrm{Cl}(11)-\mathrm{P}(4 \mathrm{~A})-\mathrm{Cl}(12)$ & $103.0(3)$ \\
\hline $\mathrm{N}(5 \mathrm{~A})-\mathrm{P}(5 \mathrm{~A})-\mathrm{N}(6 \mathrm{~A})$ & $115.6(7)$ \\
\hline $\mathrm{N}(5 \mathrm{~A})-\mathrm{P}(5 \mathrm{~A})-\mathrm{Cl}(13)$ & $111.9(6)$ \\
\hline $\mathrm{N}(6 \mathrm{~A})-\mathrm{P}(5 \mathrm{~A})-\mathrm{Cl}(13)$ & $109.5(6)$ \\
\hline $\mathrm{N}(5 \mathrm{~A})-\mathrm{P}(5 \mathrm{~A})-\mathrm{Cl}(14)$ & $106.4(7)$ \\
\hline $\mathrm{N}(6 \mathrm{~A})-\mathrm{P}(5 \mathrm{~A})-\mathrm{Cl}(14)$ & 109.1(6) \\
\hline $\mathrm{Cl}(13)-\mathrm{P}(5 \mathrm{~A})-\mathrm{Cl}(14)$ & $103.6(3)$ \\
\hline $\mathrm{N}(6 \mathrm{~A})-\mathrm{P}(6 \mathrm{~A})-\mathrm{N}(4 \mathrm{~A})$ & $113.0(8)$ \\
\hline $\mathrm{N}(6 \mathrm{~A})-\mathrm{P}(6 \mathrm{~A})-\mathrm{Cl}(15)$ & $112.8(6)$ \\
\hline
\end{tabular}




\begin{tabular}{|c|c|}
\hline $\mathrm{N}(4 \mathrm{~A})-\mathrm{P}(6 \mathrm{~A})-\mathrm{Cl}(15)$ & $107.9(5)$ \\
\hline $\mathrm{N}(6 \mathrm{~A})-\mathrm{P}(6 \mathrm{~A})-\mathrm{Cl}(16)$ & $112.8(6)$ \\
\hline $\mathrm{N}(4 \mathrm{~A})-\mathrm{P}(6 \mathrm{~A})-\mathrm{Cl}(16)$ & $105.6(5)$ \\
\hline $\mathrm{Cl}(15)-\mathrm{P}(6 \mathrm{~A})-\mathrm{Cl}(16)$ & $104.0(3)$ \\
\hline $\mathrm{P}(6 \mathrm{~A})-\mathrm{N}(4 \mathrm{~A})-\mathrm{P}(4 \mathrm{~A})$ & $123.7(7)$ \\
\hline $\mathrm{P}(4 \mathrm{~A})-\mathrm{N}(5 \mathrm{~A})-\mathrm{P}(5 \mathrm{~A})$ & $126.2(8)$ \\
\hline$P(6 A)-N(6 A)-P(5 A)$ & $125.8(9)$ \\
\hline $\mathrm{N}(4 \mathrm{~B})-\mathrm{P}(4 \mathrm{~B})-\mathrm{N}(5 \mathrm{~B})$ & $114.4(7)$ \\
\hline $\mathrm{N}(4 \mathrm{~B})-\mathrm{P}(4 \mathrm{~B})-\mathrm{Cl}(17)$ & $113.2(6)$ \\
\hline $\mathrm{N}(5 \mathrm{~B})-\mathrm{P}(4 \mathrm{~B})-\mathrm{Cl}(17)$ & $106.0(5)$ \\
\hline $\mathrm{N}(4 \mathrm{~B})-\mathrm{P}(4 \mathrm{~B})-\mathrm{Cl}(18)$ & $112.0(5)$ \\
\hline $\mathrm{N}(5 \mathrm{~B})-\mathrm{P}(4 \mathrm{~B})-\mathrm{Cl}(18)$ & $106.6(6)$ \\
\hline $\mathrm{Cl}(17)-\mathrm{P}(4 \mathrm{~B})-\mathrm{Cl}(18)$ & $103.9(3)$ \\
\hline $\mathrm{N}(6 \mathrm{~B})-\mathrm{P}(5 \mathrm{~B})-\mathrm{N}(5 \mathrm{~B})$ & $112.7(8)$ \\
\hline $\mathrm{N}(6 \mathrm{~B})-\mathrm{P}(5 \mathrm{~B})-\mathrm{Cl}(19)$ & $113.8(6)$ \\
\hline $\mathrm{N}(5 \mathrm{~B})-\mathrm{P}(5 \mathrm{~B})-\mathrm{Cl}(19)$ & $106.7(5)$ \\
\hline $\mathrm{N}(6 \mathrm{~B})-\mathrm{P}(5 \mathrm{~B})-\mathrm{Cl}(20)$ & $111.1(6)$ \\
\hline $\mathrm{N}(5 \mathrm{~B})-\mathrm{P}(5 \mathrm{~B})-\mathrm{Cl}(20)$ & $107.5(6)$ \\
\hline $\mathrm{Cl}(19)-\mathrm{P}(5 \mathrm{~B})-\mathrm{Cl}(20)$ & $104.4(3)$ \\
\hline $\mathrm{N}(6 \mathrm{~B})-\mathrm{P}(6 \mathrm{~B})-\mathrm{N}(4 \mathrm{~B})$ & $114.2(7)$ \\
\hline $\mathrm{N}(6 \mathrm{~B})-\mathrm{P}(6 \mathrm{~B})-\mathrm{Cl}(21)$ & $111.0(6)$ \\
\hline $\mathrm{N}(4 \mathrm{~B})-\mathrm{P}(6 \mathrm{~B})-\mathrm{Cl}(21)$ & $109.1(5)$ \\
\hline $\mathrm{N}(6 \mathrm{~B})-\mathrm{P}(6 \mathrm{~B})-\mathrm{Cl}(22)$ & $108.5(6)$ \\
\hline
\end{tabular}




\begin{tabular}{|c|c|}
\hline $\mathrm{N}(4 \mathrm{~B})-\mathrm{P}(6 \mathrm{~B})-\mathrm{Cl}(22)$ & $110.5(6)$ \\
\hline $\mathrm{Cl}(21)-\mathrm{P}(6 \mathrm{~B})-\mathrm{Cl}(22)$ & $102.9(3)$ \\
\hline $\mathrm{P}(4 \mathrm{~B})-\mathrm{N}(4 \mathrm{~B})-\mathrm{P}(6 \mathrm{~B})$ & $126.7(8)$ \\
\hline $\mathrm{P}(4 \mathrm{~B})-\mathrm{N}(5 \mathrm{~B})-\mathrm{P}(5 \mathrm{~B})$ & $122.0(8)$ \\
\hline$P(5 B)-N(6 B)-P(6 B)$ & $127.8(9)$ \\
\hline $\mathrm{Cl}(24)-\mathrm{Al}(2 \mathrm{~A})-\mathrm{Cl}(23)$ & $108.9(4)$ \\
\hline $\mathrm{Cl}(24)-\mathrm{Al}(2 \mathrm{~A})-\mathrm{Cl}(25)$ & $108.3(4)$ \\
\hline $\mathrm{Cl}(23)-\mathrm{Al}(2 \mathrm{~A})-\mathrm{Cl}(25)$ & $107.2(4)$ \\
\hline $\mathrm{Cl}(24)-\mathrm{Al}(2 \mathrm{~A})-\mathrm{Cl}(26)$ & $110.1(7)$ \\
\hline $\mathrm{Cl}(23)-\mathrm{Al}(2 \mathrm{~A})-\mathrm{Cl}(26)$ & $106.9(6)$ \\
\hline $\mathrm{Cl}(25)-\mathrm{Al}(2 \mathrm{~A})-\mathrm{Cl}(26)$ & $115.2(6)$ \\
\hline $\mathrm{Cl}(30)-\mathrm{Al}(2 \mathrm{~B})-\mathrm{Cl}(27)$ & $110.6(8)$ \\
\hline $\mathrm{Cl}(30)-\mathrm{Al}(2 \mathrm{~B})-\mathrm{Cl}(28)$ & $103.3(6)$ \\
\hline $\mathrm{Cl}(27)-\mathrm{Al}(2 \mathrm{~B})-\mathrm{Cl}(28)$ & $108.1(4)$ \\
\hline $\mathrm{Cl}(30)-\mathrm{Al}(2 \mathrm{~B})-\mathrm{Cl}(29)$ & $116.8(5)$ \\
\hline $\mathrm{Cl}(27)-\mathrm{Al}(2 \mathrm{~B})-\mathrm{Cl}(29)$ & $108.9(4)$ \\
\hline $\mathrm{Cl}(28)-\mathrm{Al}(2 \mathrm{~B})-\mathrm{Cl}(29)$ & $108.6(4)$ \\
\hline
\end{tabular}


Table 4. Anisotropic displacement parameters $\left(\AA^{2} \times 10^{3}\right)$ for orthorhombic $\left[\mathrm{PCl}_{2} \mathrm{~N}\right]_{3} \cdot \mathrm{HAICl}_{4}$. The anisotropic displacement factor exponent takes the form: $-2 \pi^{2}\left[h^{2} a^{* 2} U^{11}+\ldots+2 h k a^{*} b^{*} U^{12}\right]$

\begin{tabular}{|c|c|c|c|c|c|c|}
\hline & $\mathrm{U}^{11}$ & $\mathrm{U}^{22}$ & $\mathrm{U}^{33}$ & $\mathrm{U}^{23}$ & $\mathrm{U}^{13}$ & $\mathrm{U}^{12}$ \\
\hline $\mathrm{Cl}(1)$ & $32(1)$ & $28(1)$ & $18(1)$ & $1(1)$ & $2(1)$ & $3(1)$ \\
\hline $\mathrm{Cl}(2)$ & $33(1)$ & $31(1)$ & $20(1)$ & 1(1) & $-5(1)$ & $-3(1)$ \\
\hline $\mathrm{Cl}(3)$ & $39(1)$ & $28(1)$ & $29(1)$ & $8(1)$ & $-4(1)$ & $7(1)$ \\
\hline $\mathrm{Cl}(4)$ & $42(1)$ & $26(1)$ & $26(1)$ & $4(1)$ & $3(1)$ & $-6(1)$ \\
\hline $\mathrm{Cl}(5)$ & $66(2)$ & $26(1)$ & $36(1)$ & $5(1)$ & $-20(1)$ & $-15(1)$ \\
\hline $\mathrm{Cl}(6)$ & $66(1)$ & $30(1)$ & $31(1)$ & $-7(1)$ & $6(1)$ & $18(1)$ \\
\hline $\mathrm{P}(1)$ & $31(1)$ & $19(1)$ & $13(1)$ & $1(1)$ & $-1(1)$ & $-3(1)$ \\
\hline $\mathrm{P}(2)$ & $32(1)$ & $14(1)$ & $15(1)$ & 1(1) & $-1(1)$ & $0(1)$ \\
\hline $\mathrm{P}(3)$ & $45(1)$ & $14(1)$ & $14(1)$ & $0(1)$ & $-2(1)$ & $3(1)$ \\
\hline $\mathrm{N}(1)$ & $38(3)$ & $17(2)$ & $16(2)$ & $3(2)$ & $1(2)$ & $-4(3)$ \\
\hline $\mathrm{N}(2)$ & $37(3)$ & $15(2)$ & $21(2)$ & $3(2)$ & $-3(3)$ & $-2(3)$ \\
\hline $\mathrm{N}(3)$ & $33(3)$ & $16(2)$ & $17(2)$ & $-3(2)$ & $0(3)$ & $-3(3)$ \\
\hline $\mathrm{Cl}(7)$ & $64(1)$ & $39(1)$ & $30(1)$ & $-3(1)$ & $-16(1)$ & $-9(1)$ \\
\hline $\mathrm{Cl}(8)$ & $90(2)$ & $17(1)$ & $26(1)$ & $4(1)$ & $-3(1)$ & $-3(1)$ \\
\hline $\mathrm{Cl}(9)$ & $75(1)$ & 19(1) & $26(1)$ & $-7(1)$ & $-7(1)$ & $0(1)$ \\
\hline $\mathrm{Cl}(10)$ & $56(1)$ & $44(1)$ & $33(1)$ & $3(1)$ & $3(1)$ & $5(1)$ \\
\hline $\mathrm{Al}(1)$ & $57(1)$ & 19(1) & $15(1)$ & $-2(1)$ & $-4(1)$ & $-2(1)$ \\
\hline $\mathrm{Cl}(11)$ & $36(2)$ & $36(2)$ & $33(2)$ & $-12(2)$ & $-3(2)$ & $4(2)$ \\
\hline
\end{tabular}




\begin{tabular}{|c|c|c|c|c|c|c|}
\hline $\mathrm{Cl}(12)$ & $28(2)$ & $34(2)$ & $21(2)$ & $4(2)$ & $-7(2)$ & $6(2)$ \\
\hline $\mathrm{Cl}(13)$ & $24(2)$ & $29(2)$ & $28(2)$ & 2(2) & $-8(2)$ & $0(2)$ \\
\hline $\mathrm{Cl}(14)$ & $27(2)$ & $18(2)$ & $28(2)$ & $-1(2)$ & $-1(2)$ & $-7(2)$ \\
\hline $\mathrm{Cl}(15)$ & $24(2)$ & $36(2)$ & $32(2)$ & 11(2) & $-2(2)$ & $-12(2)$ \\
\hline $\mathrm{Cl}(16)$ & $37(2)$ & $27(2)$ & $31(2)$ & $-2(2)$ & $-9(2)$ & $6(2)$ \\
\hline $\mathrm{P}(4 \mathrm{~A})$ & 21(2) & $27(2)$ & $15(2)$ & $-6(1)$ & $-2(1)$ & 1(1) \\
\hline $\mathrm{P}(5 \mathrm{~A})$ & $14(2)$ & $28(2)$ & $17(2)$ & $-1(2)$ & $-4(2)$ & 1(2) \\
\hline $\mathrm{P}(6 \mathrm{~A})$ & $17(2)$ & $20(2)$ & $18(2)$ & $3(2)$ & $-1(2)$ & $7(2)$ \\
\hline $\mathrm{N}(4 \mathrm{~A})$ & $10(6)$ & $21(6)$ & $20(7)$ & $-13(5)$ & $7(5)$ & $-8(4)$ \\
\hline $\mathrm{N}(5 \mathrm{~A})$ & $18(6)$ & $45(7)$ & $28(6)$ & $-23(6)$ & $6(5)$ & $0(6)$ \\
\hline $\mathrm{Cl}(17)$ & $34(2)$ & $35(2)$ & $37(2)$ & $-18(2)$ & $-2(2)$ & $-3(2)$ \\
\hline $\mathrm{Cl}(18)$ & $22(2)$ & $46(3)$ & $17(2)$ & $0(2)$ & $-5(2)$ & 11(3) \\
\hline $\mathrm{Cl}(19)$ & $24(2)$ & $28(2)$ & $24(2)$ & $4(2)$ & $-4(2)$ & $5(2)$ \\
\hline $\mathrm{Cl}(20)$ & $27(2)$ & 23(3) & 33(3) & $-6(2)$ & $1(2)$ & $-10(2)$ \\
\hline $\mathrm{Cl}(21)$ & $17(2)$ & $22(2)$ & $29(2)$ & $2(2)$ & $7(1)$ & $-1(2)$ \\
\hline $\mathrm{Cl}(22)$ & $31(2)$ & $24(2)$ & $28(2)$ & $4(2)$ & $-1(2)$ & $4(2)$ \\
\hline $\mathrm{P}(4 \mathrm{~B})$ & $14(2)$ & $34(2)$ & $18(2)$ & $-8(2)$ & $2(2)$ & 1(1) \\
\hline $\mathrm{P}(5 \mathrm{~B})$ & $8(2)$ & $24(2)$ & $15(2)$ & $-2(2)$ & $-1(2)$ & $5(2)$ \\
\hline $\mathrm{P}(6 \mathrm{~B})$ & $19(2)$ & $17(2)$ & $13(2)$ & 1(1) & $5(2)$ & $3(2)$ \\
\hline $\mathrm{N}(4 \mathrm{~B})$ & $14(6)$ & $30(6)$ & $16(5)$ & $17(5)$ & $-1(5)$ & $-4(6)$ \\
\hline $\mathrm{N}(5 \mathrm{~B})$ & $22(7)$ & $45(9)$ & $10(6)$ & $-2(5)$ & $1(5)$ & $6(6)$ \\
\hline $\mathrm{Cl}(23)$ & $43(2)$ & 21(2) & $35(2)$ & $2(2)$ & $-15(2)$ & 2(2) \\
\hline $\mathrm{Cl}(24)$ & $26(2)$ & $24(2)$ & 71(3) & 11(2) & $-6(2)$ & $-10(1)$ \\
\hline
\end{tabular}




\begin{tabular}{lllllll}
$\mathrm{Cl}(25)$ & $22(2)$ & $31(2)$ & $26(2)$ & $-3(1)$ & $-4(1)$ & $-4(1)$ \\
$\mathrm{Cl}(26)$ & $42(7)$ & $45(4)$ & $25(3)$ & $-14(3)$ & $6(4)$ & $10(4)$ \\
$\mathrm{Al}(2 \mathrm{~A})$ & $21(3)$ & $22(3)$ & $18(2)$ & $5(2)$ & $-7(3)$ & $2(3)$ \\
$\mathrm{Cl}(27)$ & $90(4)$ & $38(3)$ & $31(2)$ & $6(2)$ & $28(2)$ & $-24(3)$ \\
$\mathrm{Cl}(28)$ & $29(2)$ & $27(2)$ & $71(3)$ & $8(2)$ & $6(2)$ & $9(2)$ \\
$\mathrm{Cl}(29)$ & $26(2)$ & $38(2)$ & $30(2)$ & $-9(2)$ & $-2(1)$ & $8(2)$ \\
$\mathrm{Cl}(30)$ & $40(7)$ & $54(7)$ & $24(4)$ & $-11(4)$ & $4(4)$ & $-5(5)$ \\
$\mathrm{Al}(2 \mathrm{~B})$ & $25(4)$ & $21(3)$ & $25(3)$ & $-3(2)$ & $4(4)$ & $12(4)$ \\
& & & & & \\
\hline
\end{tabular}




\section{The reactions of $\left[\mathrm{PCl}_{2} \mathrm{~N}\right]_{3}$ with $\mathrm{HX}$ (experimental procedures and NMR data)}

\section{Synthesis and purification of $\mathrm{HX}(\mathrm{X}=\mathrm{Cl}$ or $\mathrm{Br})$.}

In the glove box, $\mathrm{AlX}_{3}\left(\mathrm{AlBr}_{3}=0.905 \mathrm{~g}, 3.39 \mathrm{mmol}, \mathrm{AlCl}_{3}=0.453 \mathrm{~g}, 3.39 \mathrm{mmol}\right)$ was added to a Schlenk flask and a liquid addition funnel containing an excess ( $\sim 5.0 \mathrm{~mL})$ of $\mathrm{H}_{2} \mathrm{O}$ was attached to the Schlenk flask. The Schlenk flask was attached to a high vacuum line and evacuated. The liquid addition funnel was opened and $\mathrm{H}_{2} \mathrm{O}$ was slowly added to the Schlenk flask to produce $\mathrm{HX}(\mathrm{g})$. The $\mathrm{HX}(\mathrm{g})$ was pumped through 4 consecutive traps submerged in cold baths of $0{ }^{\circ} \mathrm{C},-78{ }^{\circ} \mathrm{C},-196{ }^{\circ} \mathrm{C}$ and $-196{ }^{\circ} \mathrm{C}$ respectively. The gaseous products were trapped in the $-196{ }^{\circ} \mathrm{C}$ cold baths, were transferred into a gas bulb and stored until used. IR spectra were obtained to confirm the purity of HX.

\section{Attempted protonation of $\left[\mathrm{PCl}_{2} \mathrm{~N}\right]_{3}$ with $\mathrm{HX}(\mathrm{X}=\mathrm{Cl}$ or $\mathrm{Br})$.}

In a thick-walled NMR tube (I.D. $=3 \mathrm{~mm}$ and O.D. $=5 \mathrm{~mm}),\left[\mathrm{PCl}_{2} \mathrm{~N}\right]_{3}(20 \mathrm{mg}, 0.058 \mathrm{mmol})$ was added to $\mathrm{CDCl}_{3}$. The NMR attached to the high vacuum line, degassed and $\sim 1.5 \mathrm{~atm}$ of $\mathrm{HX}(\mathrm{g})(0.092 \mathrm{mmol})$ was condensed in. The NMR tube was flame-sealed and spectra were obtained (Figures 1 and 2 below). 


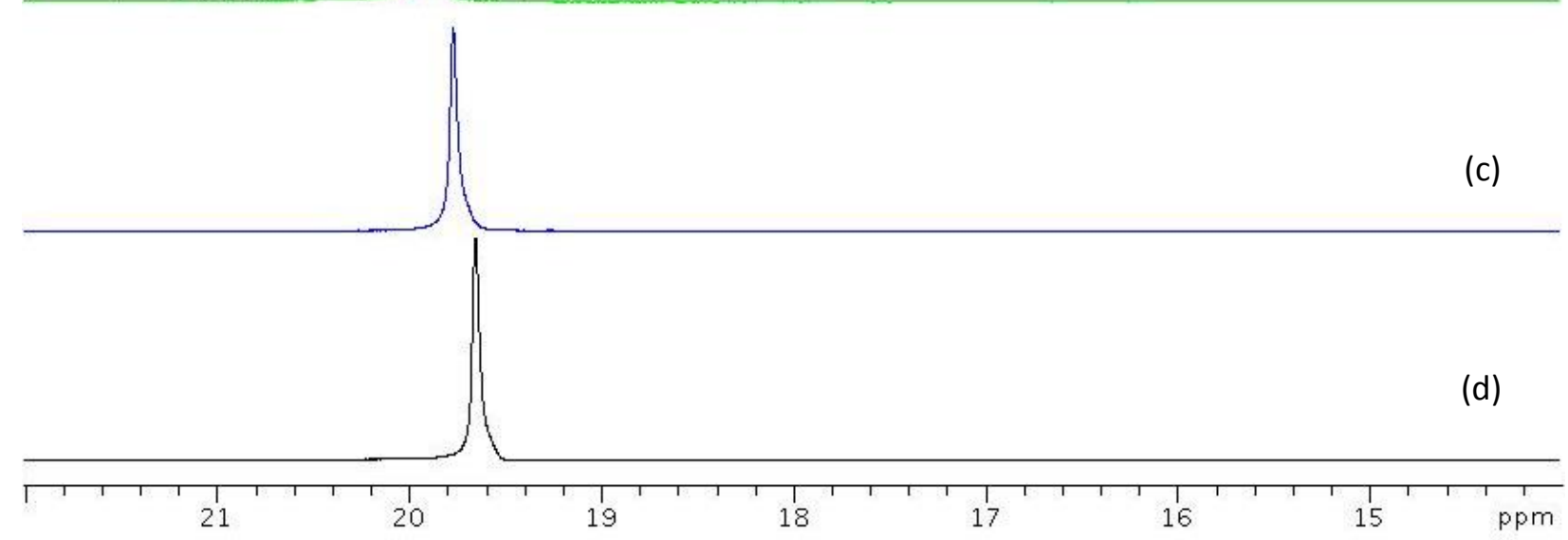

Figure $1 .{ }^{31} \mathrm{P}$ VT NMR spectra of a mixture of $\left[\mathrm{PCl}_{2} \mathrm{~N}\right]_{3}$ and $\sim 1.5$ atm $\mathrm{HBr}$ in $\mathrm{CDCl}_{3}$ taken between -40 and $25^{\circ} \mathrm{C}$. (a) $25^{\circ} \mathrm{C}$, (b) $0^{\circ} \mathrm{C}$, (c) $-20^{\circ} \mathrm{C}$ and (d) $-40^{\circ} \mathrm{C}$.

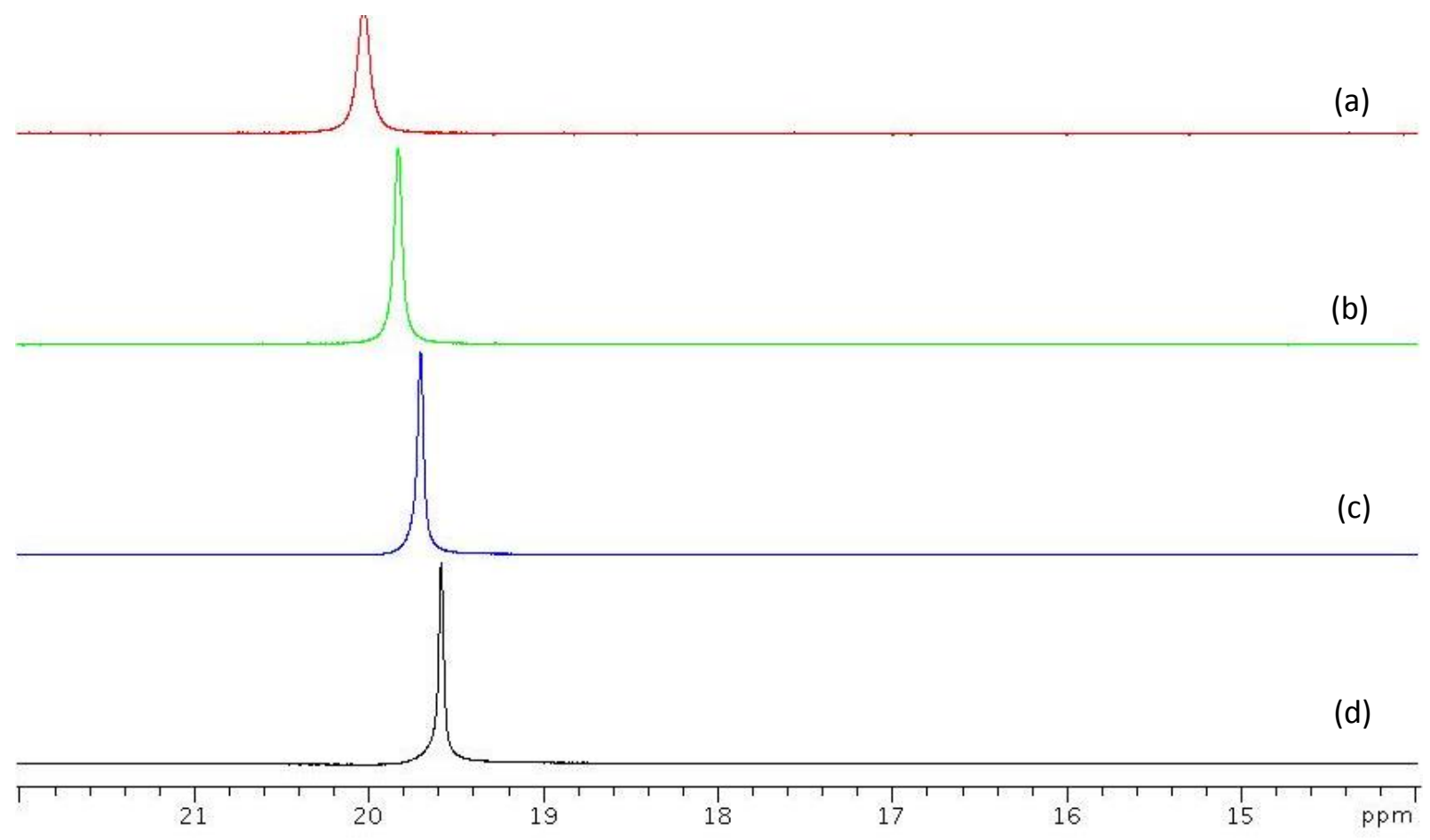

Figure 2. ${ }^{31} \mathrm{P}$ VT NMR spectra of a mixture of $\left[\mathrm{PCl}_{2} \mathrm{~N}\right]_{3}$ and $\sim 1.5 \mathrm{~atm} \mathrm{HCl}$ in $\mathrm{CDCl}_{3}$ taken between -40 and $25^{\circ} \mathrm{C}$. (a) $25^{\circ} \mathrm{C}$, (b) $0{ }^{\circ} \mathrm{C}$, (c) $-20^{\circ} \mathrm{C}$ and (d) $-40^{\circ} \mathrm{C}$. 
(a)

(b)

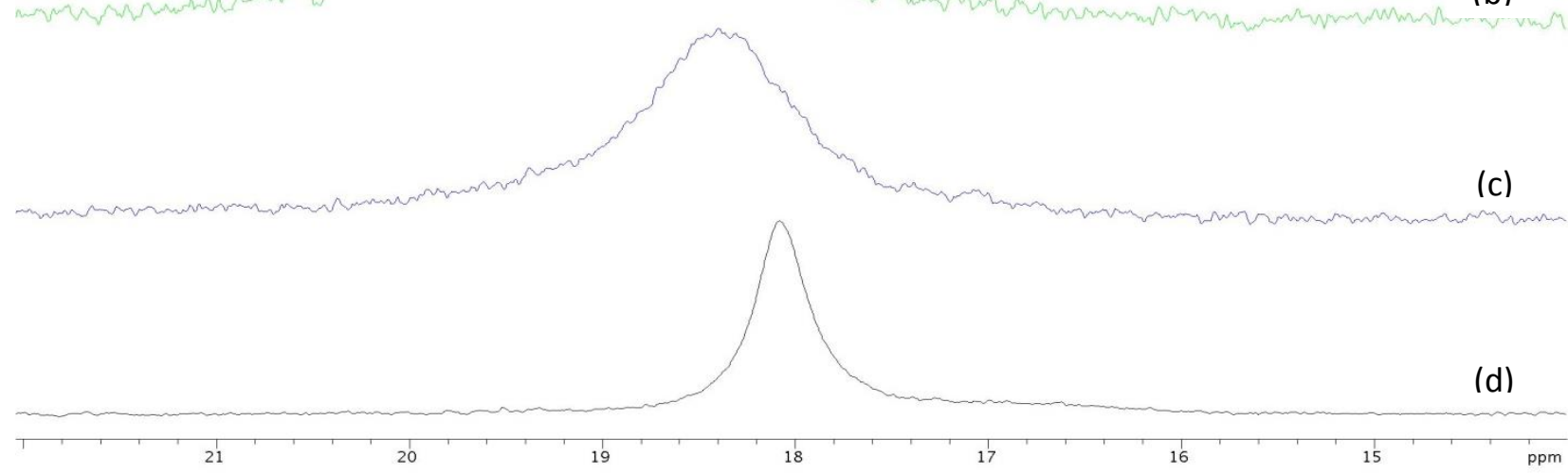

Figure 3: ${ }^{31} \mathrm{P}$ VT NMR spectra $\left[\mathrm{PCl}_{2} \mathrm{~N}\right]_{3} \bullet \mathrm{HGaCl}_{4}$ in $\mathrm{CDCl}_{3}$ taken between -40 and $25^{\circ} \mathrm{C}$. (a) $25^{\circ} \mathrm{C}$, (b) 0 ${ }^{\circ} \mathrm{C},(\mathrm{c})-20{ }^{\circ} \mathrm{C}$ and $(\mathrm{d})-40{ }^{\circ} \mathrm{C}$.

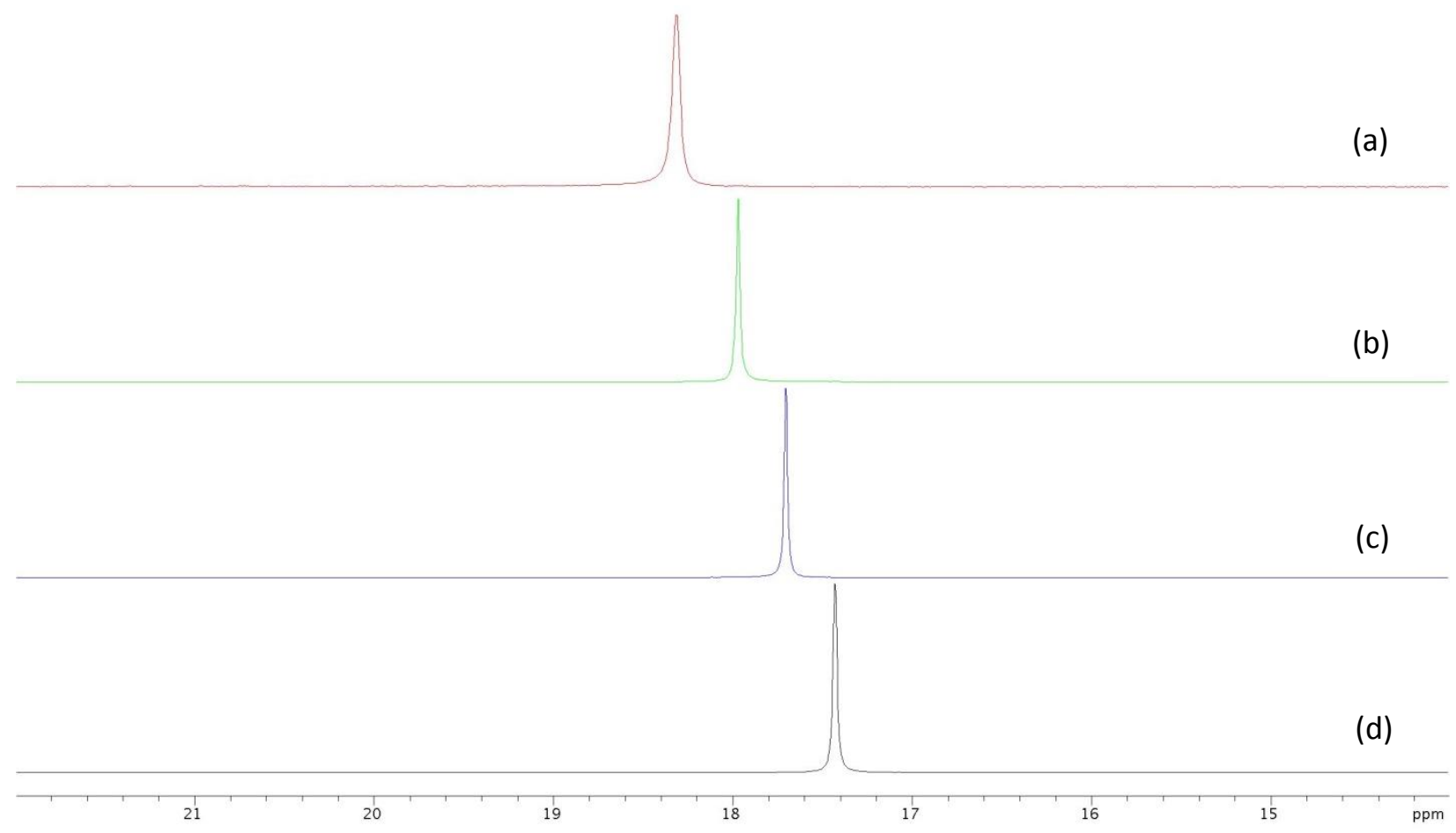

Figure 4: ${ }^{31} \mathrm{P}$ VT NMR spectra $\left[\mathrm{PCl}_{2} \mathrm{~N}\right]_{3} \cdot \mathrm{HAlBr}_{4}$ in $\mathrm{CDCl}_{3}$ taken between -40 and $25^{\circ} \mathrm{C}$. (a) $25{ }^{\circ} \mathrm{C}$, (b) 0 ${ }^{\circ} \mathrm{C}$, (c) $-20{ }^{\circ} \mathrm{C}$ and (d) $-40{ }^{\circ} \mathrm{C}$. 


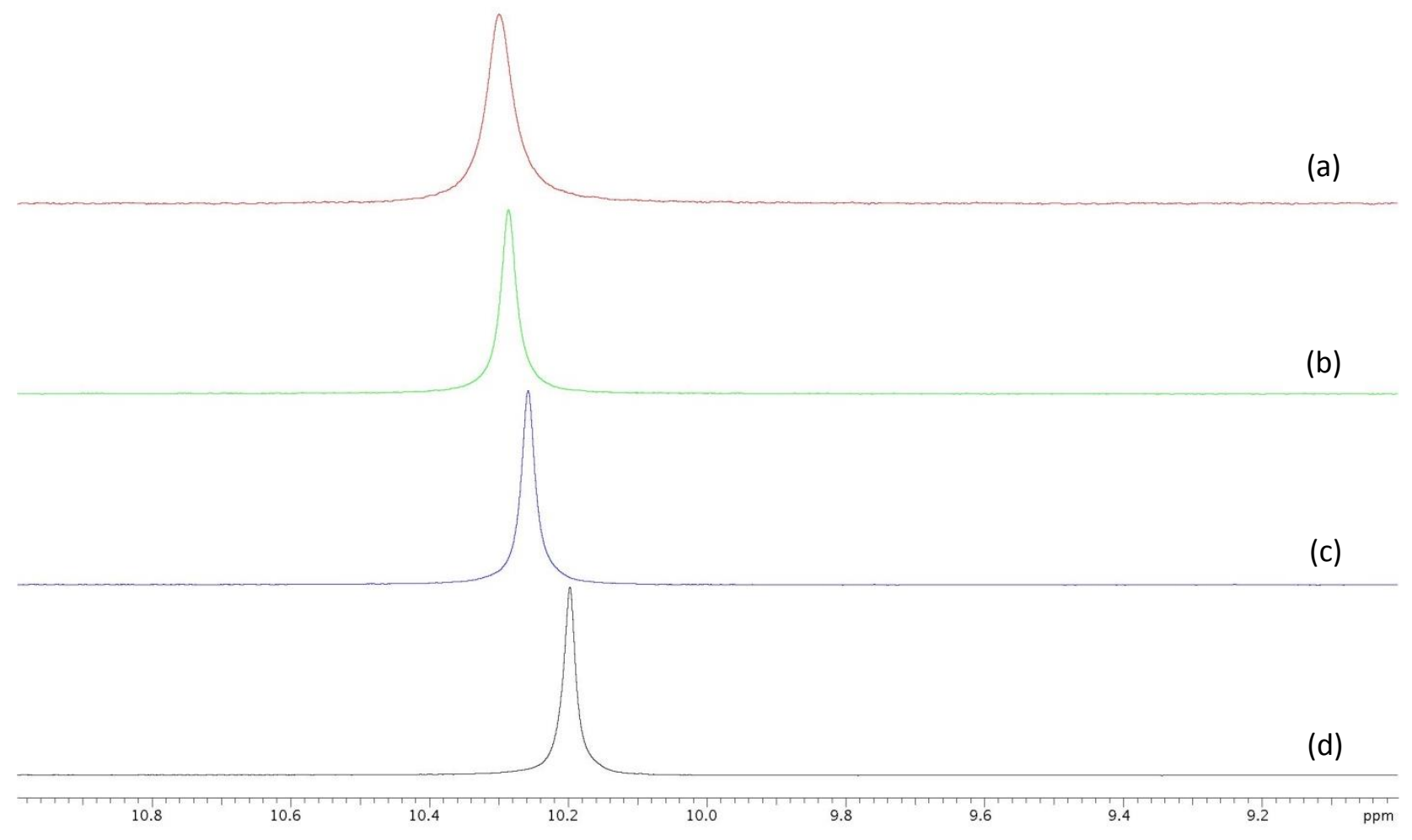

Figure 5: ${ }^{1} \mathrm{H}$ VT NMR spectra $\left[\mathrm{PCl}_{2} \mathrm{~N}\right]_{3} \cdot \mathrm{HGaCl}_{4}$ in $\mathrm{CDCl}_{3}$ taken between -40 and $25^{\circ} \mathrm{C}$. (a) $25^{\circ} \mathrm{C}$, (b) $0{ }^{\circ} \mathrm{C}$, (c) $-20^{\circ} \mathrm{C}$ and (d) $-40{ }^{\circ} \mathrm{C}$.

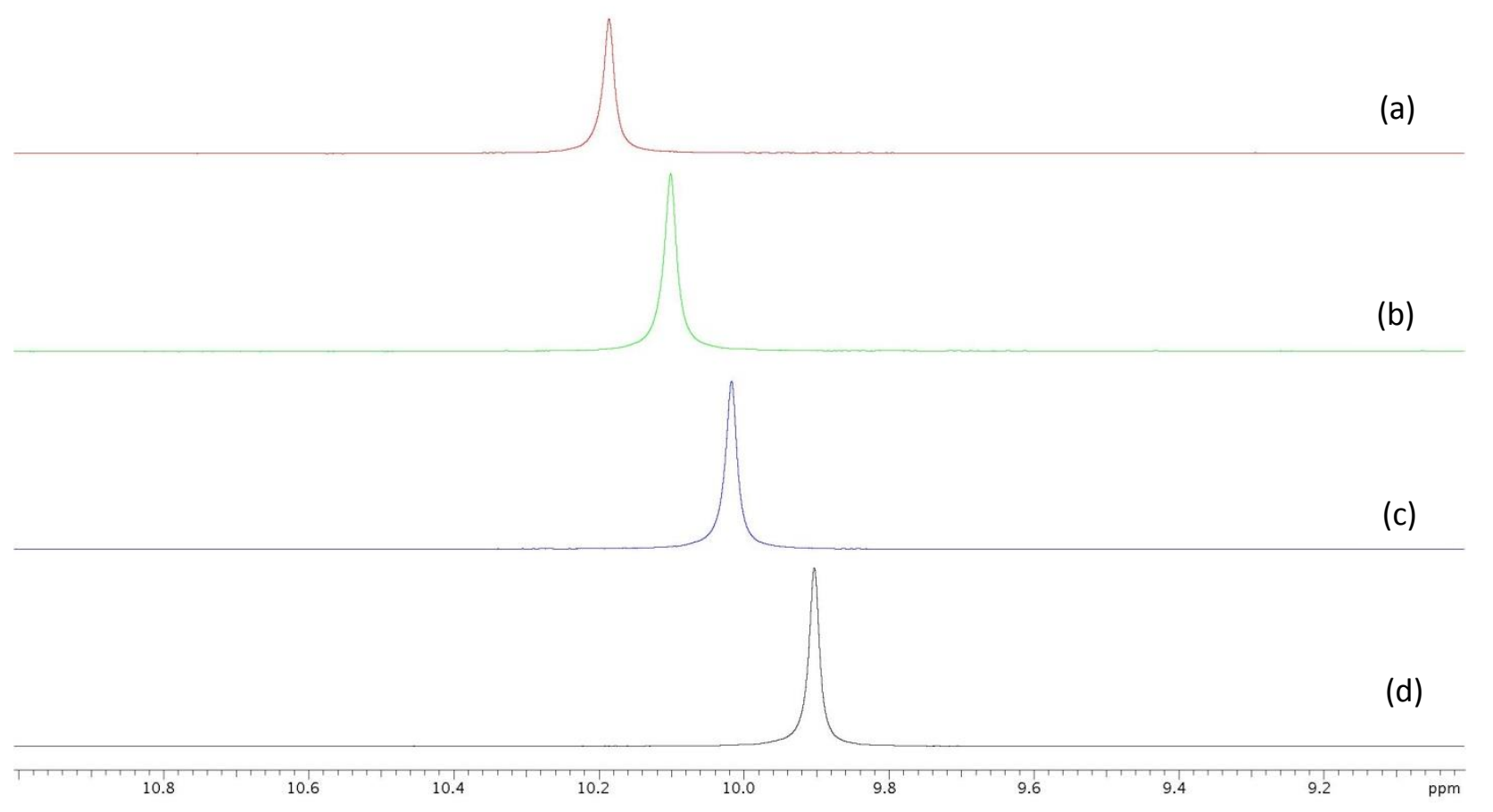

Figure 6. ${ }^{1} \mathrm{H}$ VT NMR spectra of $\left[\mathrm{PCl}_{2} \mathrm{~N}\right]_{3} \cdot \mathrm{HAlBr}_{4}$ in $\mathrm{CDCl}_{3}$ taken between -40 and $25^{\circ} \mathrm{C}$. (a) $25^{\circ} \mathrm{C}$, (b) $0{ }^{\circ} \mathrm{C}$, (c) $-20^{\circ} \mathrm{C}$ and (d) $-40^{\circ} \mathrm{C}$. 


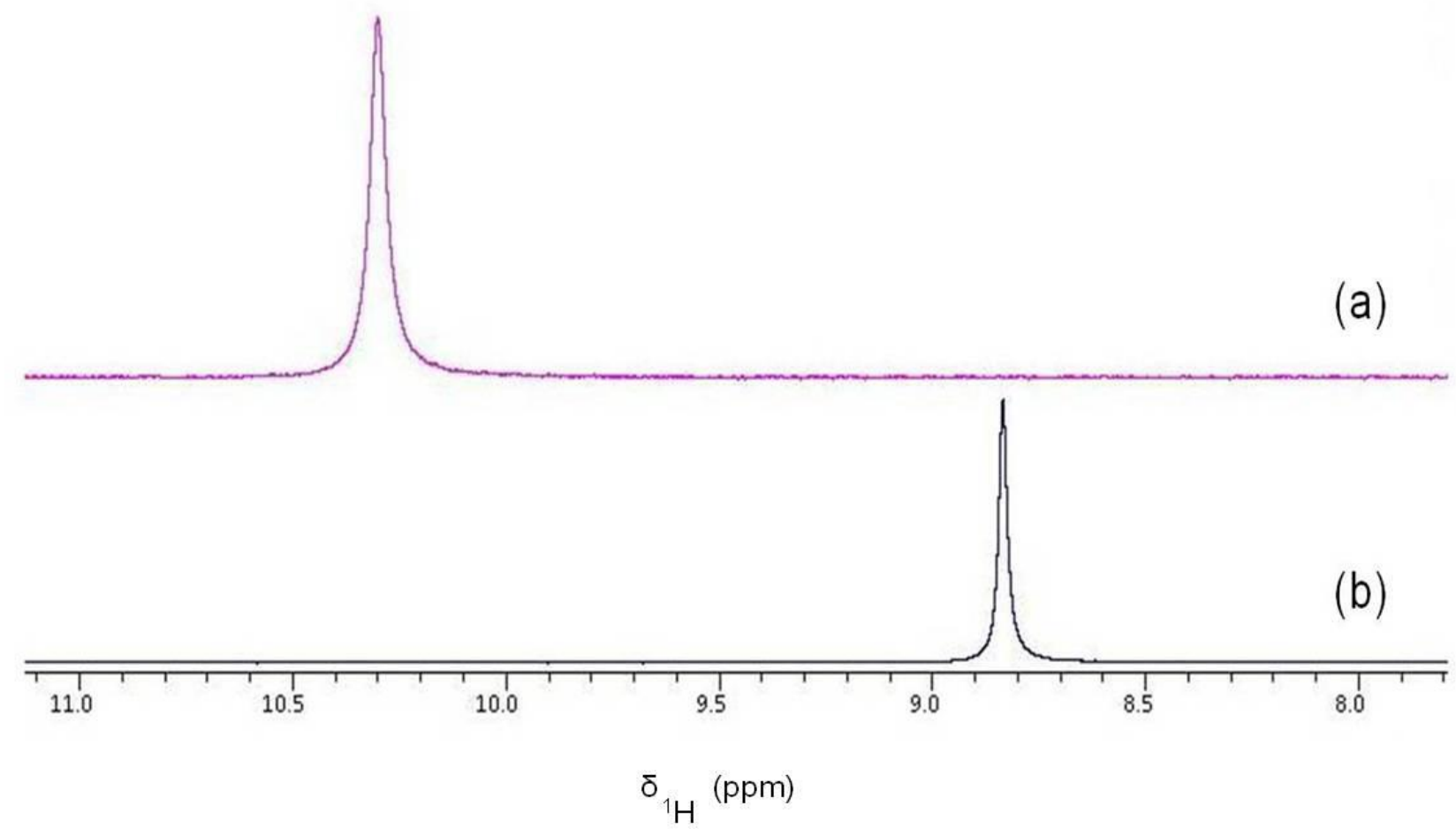

Figure 7. ${ }^{1} \mathrm{H}$ NMR spectra of $\left[\mathrm{PCl}_{2} \mathrm{~N}\right]_{3} \cdot \mathrm{HGaCl}_{4}$ in $\mathrm{CDCl}_{3}$ taken at $30{ }^{\circ} \mathrm{C}$ to investigate the effect of additional $\mathrm{GaCl}_{3}$ : (a) sample of $\left[\mathrm{PCl}_{2} \mathrm{~N}\right]_{3} \cdot \mathrm{HGaCl}_{4}$, (b) the same sample of $\left[\mathrm{PCl}_{2} \mathrm{~N}\right]_{3} \cdot \mathrm{HGaCl}_{4}$ with additional $\mathrm{GaCl}_{3}$.

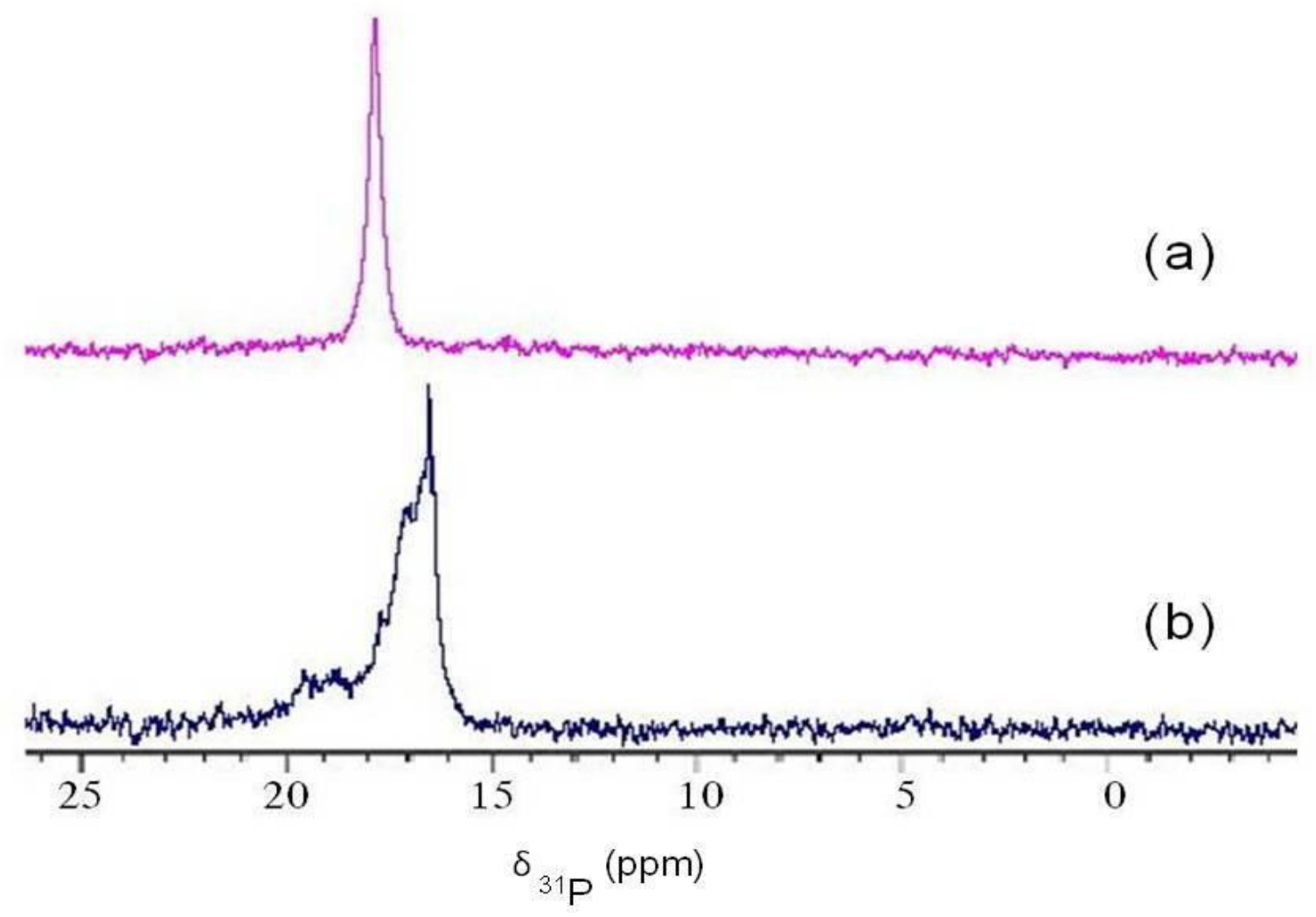

Figure 8. ${ }^{31} \mathrm{P} \mathrm{NMR}$ spectra of $\left[\mathrm{PCl}_{2} \mathrm{~N}\right]_{3} \cdot \mathrm{HGaCl}_{4}$ in $\mathrm{CDCl}_{3}$ taken at $30{ }^{\circ} \mathrm{C}$ to investigate the effect of additional $\mathrm{GaCl}_{3}$ : (a) sample of $\left[\mathrm{PCl}_{2} \mathrm{~N}_{3}{ }_{3} \mathrm{HGaCl}_{4}\right.$ (b) the same sample of $\left[\mathrm{PCl}_{2} \mathrm{~N}_{3} \cdot \mathrm{HGaCl}_{4}\right.$ with additional $\mathrm{GaCl}_{3}$. 


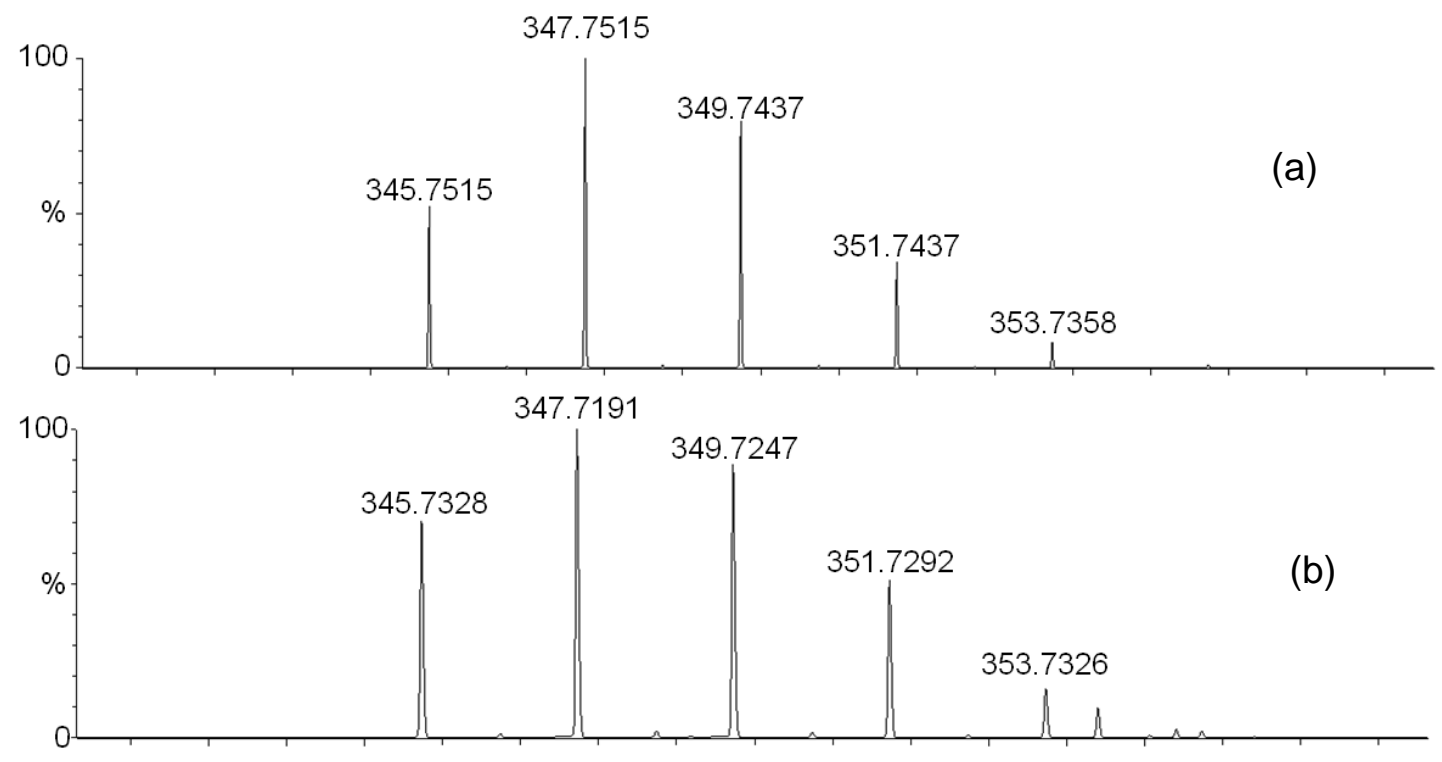

Figure 9. ESI Mass spectrum of $\left[\mathrm{PCl}_{2} \mathrm{~N}\right]_{3} \mathrm{HGaCl}_{4}$ in positive mode. (a) theoretical isotope distribution for $\left(\mathrm{H}\left[\mathrm{PCl}_{2} \mathrm{~N}\right]_{3}\right)^{+}$, (b) experimental isotope distribution.

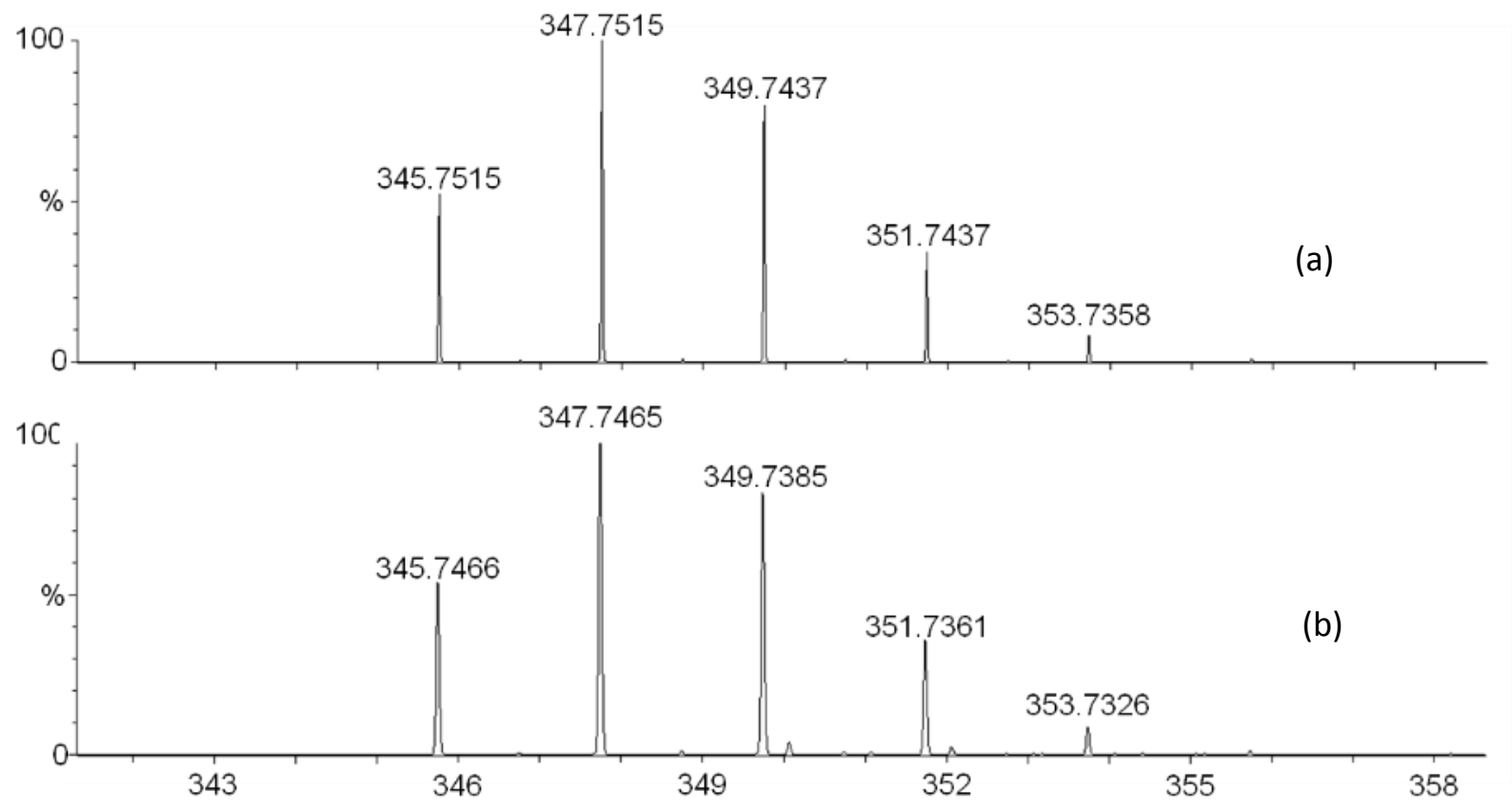

Figure 10. ESI Mass spectrum of $\left[\mathrm{PCl}_{2} \mathrm{~N}\right]_{3} \cdot \mathrm{HAlBr}_{4}$ in positive mode. (a) theoretical isotope distribution for $\left(\mathrm{H}\left[\mathrm{PCl}_{2} \mathrm{~N}\right]_{3}\right)^{+}$, (b) experimental isotope distribution. 


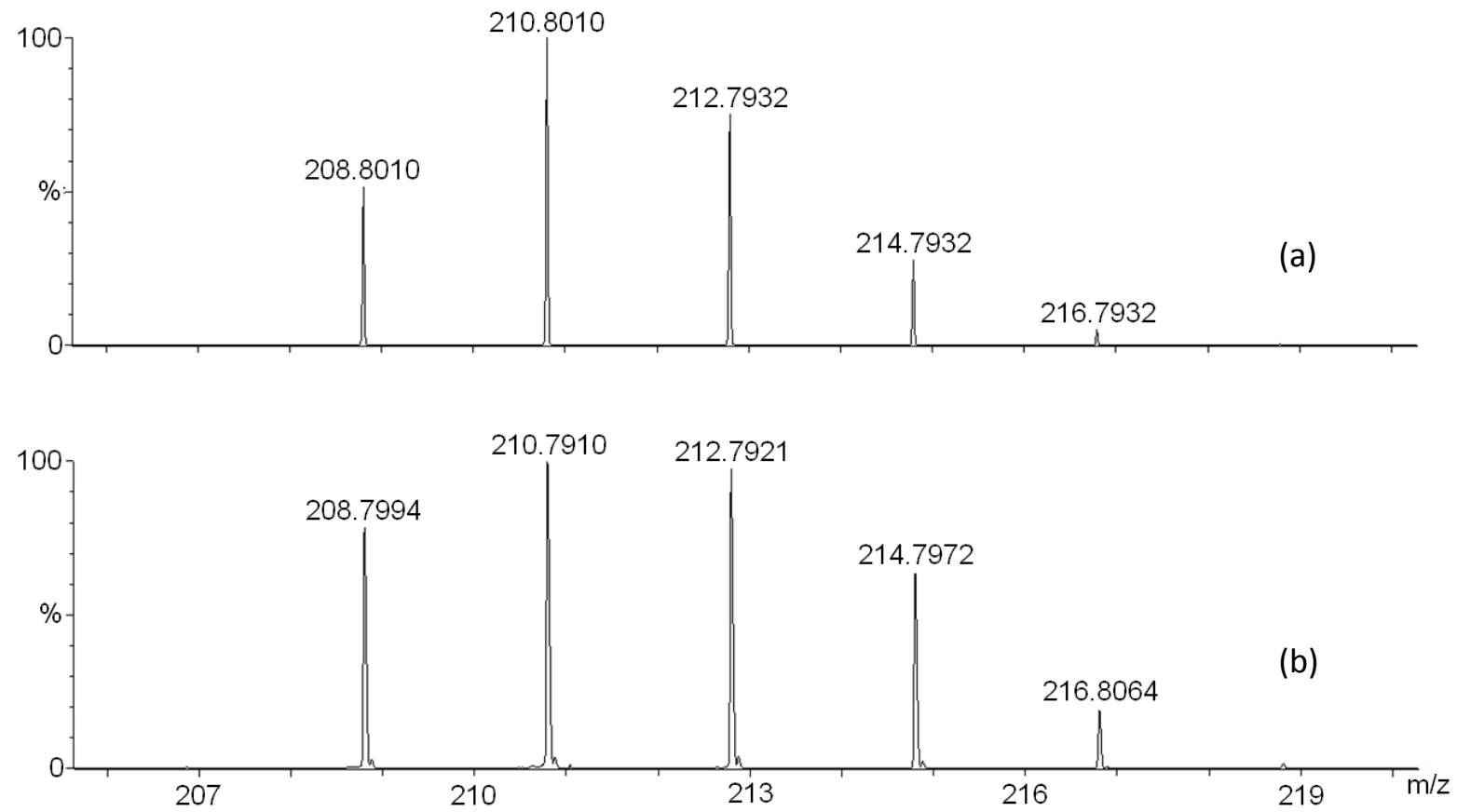

Figure 11. ESI Mass spectrum of $\left[\mathrm{PCl}_{2} \mathrm{~N}\right]_{3} \cdot \mathrm{HGaCl}_{4}$ in negative mode. (a) theoretical isotope distribution for $\mathrm{GaCl}_{4}^{-}$, (b) experimental isotope distribution. 